Project 09-837

\title{
Chalcogenide Glass Radiation Sensor; Materials Development, Design and Device Testing
}

\section{Integrated University Programs}

Dr. Maria Mitkova

Boise State University

In collaboration with:

Arizona State University

Mark Mullen, Technical POC

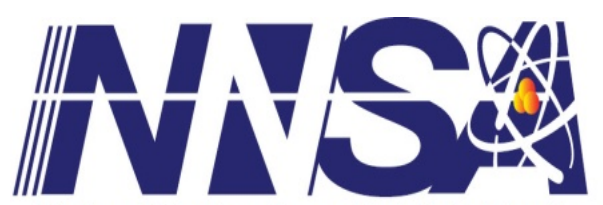


Project Title: Chalcogenide Glass Radiation Sensor; Materials Development, Design and Device Testing

Covering Period: Time period covered by the report (Nov, 2009 through March 31, 2013)

Date of Report: $\quad$ Date (April 30. 2013)

Recipient: $\quad$ Boise State University College of Engineering

Address: 1910 University Dr., Boise, ID 83725-2075

Award Number: DE-AC07-05ID14517 task order 00027 under Blanket Master Contract No 00041394

Project Number: $\quad 09-837$

Principal Maria Mitkova

Investigator:, tel. (208) 426 1319, e-mail: mariamitkovba@boisestate.edu

Collaborators: $\quad$ Darryl P. Butt, e-mail: DarrylButt@boisestate.edu

Michael N. Kozicki e-mail: Michael.Kozicki@asu.edu

Hugh Barnaby e-mail: hbarnaby@asu.edu

Project Objective: Development of a low cost, high performance microelectronic device that reacts to $\gamma$ radiation to produce an easily measured change in electrical resistance. This radiation sensor is a two-terminal micro device with an active region consisting of a chalcogenide glass $(\mathrm{ChG})$. Exposure to ionizing radiation stimulates radiation induced effects (RIE) in the active region, which promotes silver $(\mathrm{Ag})$ diffusion and incorporation in the $\mathrm{ChG}$ thereby reducing the material's resistivity. Since these devices are based on amorphous films, they can be fabricated on flexible and non-planar substrates which will increase their range of application. This approach is characterized by completely new principles of operation that offer low power consumption, compatibility with integrated circuit fabrication, and operational reversibility which allows for calibration and reuse. Since the family of the chalcogenide glasses includes a large number of materials, there are extensive possibilities to tailor the sensitivity of the sensor to particular use situations.

Background: Citizenship:
This is the final report summarizing the work on the project.

All PIs are US citizens

The citizenship of the students and post doc working on the project is the following: 
There was one post doc - Ping Chen - he has worked at BSU who is citizen of China

One student worked on the project at BSU - Mahesh Ailavajhala who is a US citizen

At ASU one US citizen - John Sochacki has worked on the project and the post doc and other students working at ASU on it have the following citizenships:

Yago Gonzales Velo (post doc) - France

Ivan Sanchez Esqueda - Mexico

Ankita Chandaravan - India

Pradeep Dandamudy - India

\section{ABSTRACT}

For many decades, various radiation detecting material have been extensively researched, to find a better material or mechanism for radiation sensing. Recently, there is a growing need for a smaller and effective material or device that can perform similar functions of bulkier Geiger counters and other measurement options, which fail the requirement for easy, cheap and accurate radiation dose measurement. Here arises the use of thin film chalcogenide glass, which has unique properties of high thermal stability along with high sensitivity towards short wavelength radiation. The unique properties of chalcogenide glasses are attributed to the lone pair $p$-shell electrons, which provide some distinctive optical properties when compared to crystalline material. These qualities are derived from the energy band diagram and the presence of localized states in the band gap. Chalcogenide glasses have band tail states and localized states, along with the two band states. These extra states are primarily due to the lone pair electrons as well as the amorphous structure of the glasses. The localized states between the conductance band (CB) and valence band (VB) are primarily due to the presence of the lone pair electrons, while the band tail states are attributed to the Van der Waal's forces between layers of atoms [1]. Localized states are trap locations within the band gap where electrons from the valence band can hop into, in their path towards the conduction band. Tail states on the other hand are locations near the band gap edges and are known as Urbach tail states $\left(\mathrm{E}_{\mathrm{u}}\right)$. These states are occupied with many electrons that can participate in the various transformations due to interaction with photons. According to Y. Utsugi et. al.[2], the electron-phonon interactions are responsible for the generation of the Urbach tails. These states are responsible for setting the absorption edge for these glasses and photons with energy near the band gap affect these states.

We have studied the effect of $\mathrm{x}$-rays and $\gamma$-rays, on thin film chalcogenide glasses and applied them in conjunction with film incorporating a silver source in a new type of radiation sensor for which we have an US patent application [3]. In this report, we give data about our studies regarding our designed radiation sensor along with the testing and performance at various radiation doses. These studies have been preceded by materials characterization research related to the compositional and structural characteristics of the active materials used in the radiation sensor design. 
During the work on the project, we collected a large volume of material since every experiment was repeated many times to verify the results. We conducted a comprehensive material research, analysis and discussion with the aim to understand the nature of the occurring effects, design different structures to harness these effects, generated models to aid in the understanding the effects, built different device structures and collected data to quantify device performance. These various aspects of our investigation have been detailed in previous quarterly reports. In this report, we present our main results and emphasize on the results pertaining to the core project goals - materials development, sensor design and testing and with an emphasis on classifying the appropriate material and design for the optimal application.

The report has three main parts: (i) Presentation of the main data; (ii) Bulleted summary of the most important results; (iii) List of the patent, journal publications, conference proceedings and conferences participation, occurring as a result of working on the project.

MATERIALS CHARACTERIZATION STUDIES

\subsection{Ge-S Chalcogenide System - Influence of $\gamma$ Radiation}

Before starting devices formation and testing, we performed detailed materials characterization to understand the manner in which radiation affects the material towards aiding functioning as a radiation-sensing device. Films consisting of bare chalcogenide films, silvertopped chalcogenide films and prototype of big-size devices were fabricated using thermal evaporation. The initial and the radiated films were studied using Raman spectroscopy, Energy Dispersive X-ray Spectroscopy (EDS) and X-ray Diffraction (XRD). The electrical performance of the devices was characterized by Current vs. Voltage (I-V) measurements. Various compositions of thin films were created using bulk glass compositions of $\mathrm{Ge}_{\mathrm{x}} \mathrm{S}_{1-\mathrm{x}}(\mathrm{x}=20,30,33$, 40). The real compositions of the obtained films were studied using EDS, which showed that the compositions were $\mathrm{Ge}_{\mathrm{x}} \mathrm{S}_{1-\mathrm{x}}(\mathrm{x}=33.4,35.1,43.0,46.1)$ respectively. Here we present data, regarding the structural changes caused by different doses of $\gamma$ radiation observed in the various bare Ge-S chalcogenide systems using Raman spectroscopy.

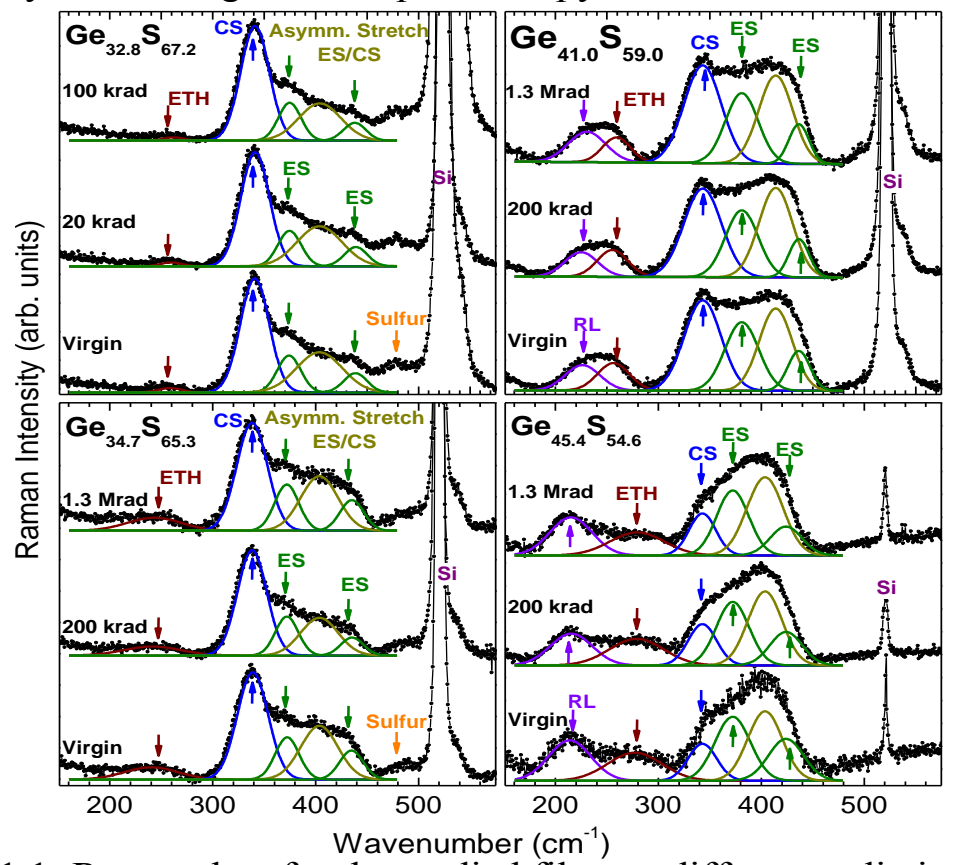

Figure 1.1: Raman data for the studied films at different radiation doses 
Raman spectra for the $\mathrm{S}$ rich glasses show a major peak located at around $340 \mathrm{~cm}^{-1}$ which represents the breathing mode of the $\mathrm{Ge}\left(\mathrm{S}_{1 / 2}\right)_{4}$ corner sharing tetrahedra in which Ge is fourfold coordinated and $\mathrm{S}$ is twofold coordinated. Its dominance fades with increasing the relative amount of Ge over 33 at.\% when the edge sharing tetrahedra, ethane-like structures and outrigger raft structure concurrently develop. It is worth mentioning that the frequency of the corner sharing mode that appears at $340 \mathrm{~cm}^{-1}$ for the $\mathrm{Ge}_{32.8} \mathrm{~S}_{67.2}$ composition, undergoes a small shift to $345 \mathrm{~cm}^{-1}$ with increase of the Ge concentration, which is derived from intertetrahedral couplings [4]. This factor is an important feature in the interaction of the glasses with radiation. The coupling with the nearby tetrahedra creates a high connectivity of the system with high concentration of S keeping it intact and the radiation at the conditions of this study does not result in significant bond breaking/occurrence that could be detected.

Based on our radiation data we suggest that for the S rich glasses, formation of electronhole pairs due to radiation is the major mechanism that is empowered by the high concentration of chalcogen atoms, which contain lone pair electrons. This creates internal electric fields produced by non-equilibrium, radiation induced effects such as formation of defect $\mathrm{C}_{1}{ }^{+}$and $\mathrm{C}_{3}{ }^{+}$ centers [5]. They are the reason for the reduction of the optical band gap reported by Xia et.al. [6] and hence increased conductivity. At the conditions of our experiments, there is no significant bond breaking and structural reorganization for chalcogen-rich glasses. However structural reorganization has been obtained for sulfur-rich $\mathrm{Ge}-\mathrm{Sb}-\mathrm{S}$ glasses that have been radiated with a 7.7 MGy dose which is much higher than used in our experiments [7].

For the glasses with 45.4 at.\% Ge, due to the reduced amount of nearby tetrahedra, restructuring of the system is possible. In this case, $\mathrm{Ge}^{2+}$ can be regarded as a modifier in the system, which contributes to breaking up the bridging sulfur. It is for this reason that radiation induces formation of a higher number of edge sharing structural units in the Ge-rich films by breaking some of the existing bonds. This has the important consequence of opening the entire structure of the films, which is shown in the figure below.
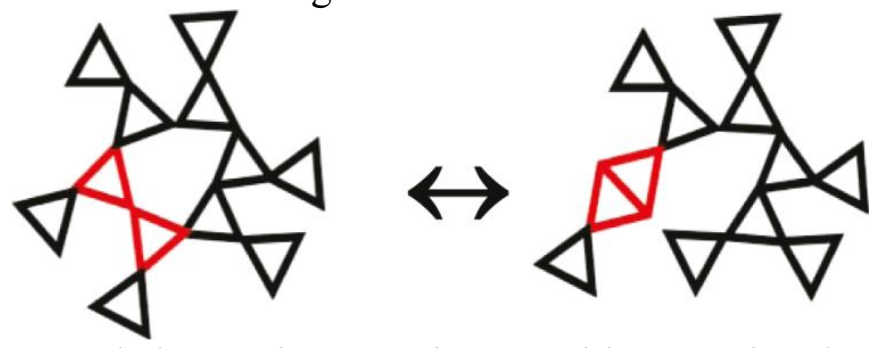

Figure 1.2: Structural change due to ES/CS transition opening the structure[7]

The increased voids and structural reorganization in addition to the electric fields effects described by O. Shpotyuk, aid in the diffusion of ionic metals such as silver, which can easily diffuse in chalcogenide glasses and form precipitates. These new precipitates were studied using Raman spectroscopy and XRD as shown below.

Comparing non-silver containing films with silver diffused films will reflect in a change in the Raman spectra, hence provides an insight to the different dynamic changes that were captured by the diffusion of silver ions within the chalcogenide structure. Observing a change in sulfur chains or other structural units, we can conclude to the formation of structural precipitates. Before explaining in detail about the diffusion products, it is important to explain how silver can diffuse within the chalcogenide film in the presence of radiation. When radiation becomes incident onto silver atoms, the radiation causes the atoms to become ionized forming $\mathrm{Ag}^{+}$ions, which are fast moving ions and due to the presence of internal fields in addition to the high 
affinity of the silver ions towards chalcogen atoms, silver diffuses and forms diffusion products with the backbone structure of the chalcogenide film. Once introduced into a non-crystalline or

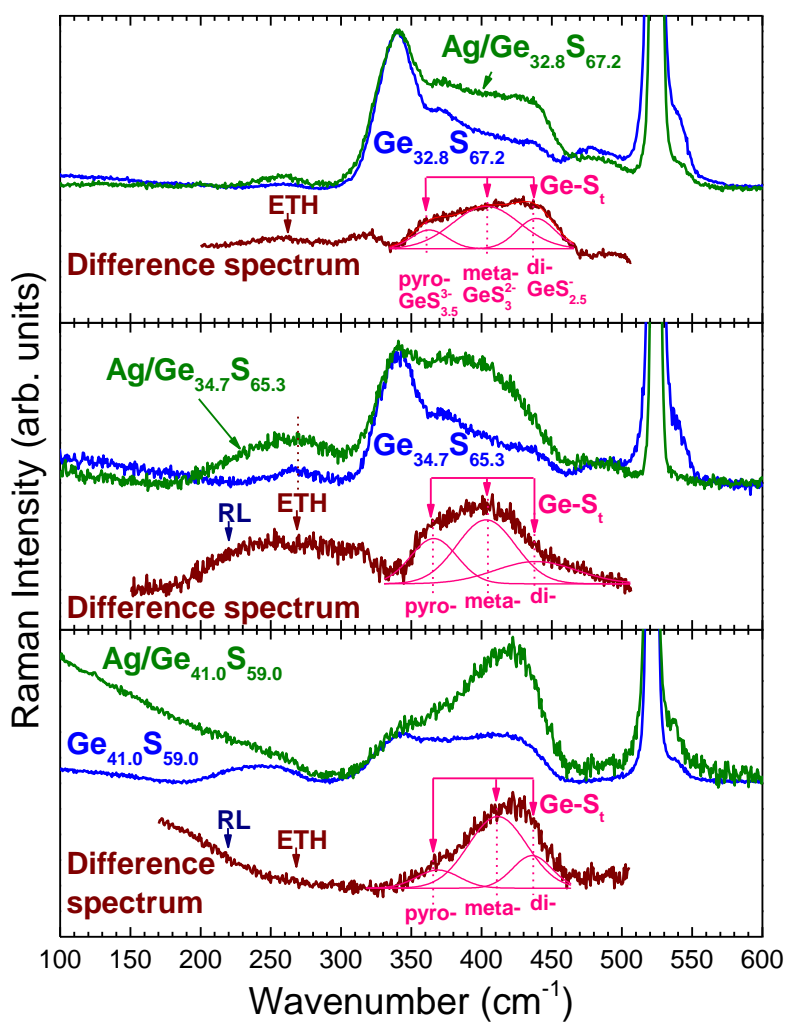

Figure 1.3: Raman Spectra of Ag containing films compared to non-Ag containing films and their difference spectra

glassy phase, Ag could form stoichiometric solids and could be included as an additive in the base network [8]. These additives can either segregate $[8,9]$ as separate phases or uniformly mix [8] with the base glass to form homogeneous solid electrolyte glasses. There are several specifics that we want to point out from the Raman Spectra of the Ag containing glasses: (i) there is intensity growth of the mode at $250 \mathrm{~cm}^{-1}$ indicating the formation of higher number of ETH structural units and significant sulfur depletion of the initial composition of the hosting structure. The number of ETH units is limited in the case of the host glass with 32.8 at.\% Ge because of the partial consumption of $\mathrm{Ag}$ atoms in formation of $\mathrm{Ag}_{2} \mathrm{~S}$. In the case of glasses with higher $\mathrm{Ge}$ concentration, the growth of this mode is better expressed because of the higher consumption of the network building blocks for Ag incorporation and the higher concentration of Ag introduced in the glass-phases (ii) The relative intensity of the mode at $343 \mathrm{~cm}^{-1}$ is reduced while the vibrations at $370 \mathrm{~cm}^{-1}$ and $400 \mathrm{~cm}^{-1}$ are strengthened. We attribute the latter modes to the development of thiogermanate bonds $\left(\mathrm{GeS}^{-}\right)$forming pyro- $\left(\mathrm{GeS}^{3-}{ }_{3.5}\right)$, meta- $\left(\mathrm{GeS}_{3}^{2-}\right)$ and di$\left(\mathrm{GeS}_{2.5}^{-}\right)$thiogermanate tetrahedra as suggested by Kamitsos at. al [9]. These various molecular structures are shown in figure below. Note the dominance of the metathiogermanate tetrahedra, which after accommodation of Ag forms the stoichiometry that is specific for this system - the $\mathrm{Ag}_{2} \mathrm{GeS}_{3}$ ternary. It is not visible on the XRD spectra because it becomes part of the amorphous network.

The fate of Ag strongly depends upon the surrounding structure of the film. As revealed by the XRD studies for the case when Ag diffuses in $\mathrm{Ge}_{32.8} \mathrm{~S}_{67.2}$ film, part of it phase separates 
and forms $\mathrm{Ag}_{2} \mathrm{~S}$ reacting with $\mathrm{S}$ from the sulfur chains and rings. However, only a small portion of $\mathrm{Ag}$ forms $\mathrm{Ag}_{2} \mathrm{~S}$ since there is significant change in the Raman Spectrum, which correlates to $\mathrm{Ag}$ becoming part of the structure rather than phase separating to form $\mathrm{Ag}_{2} \mathrm{~S}$.

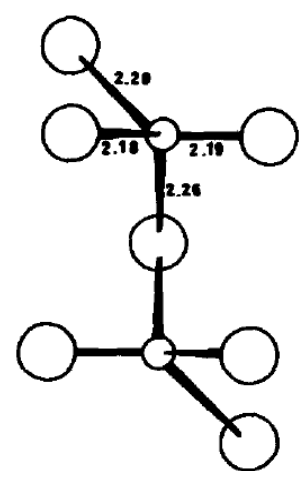

(a)

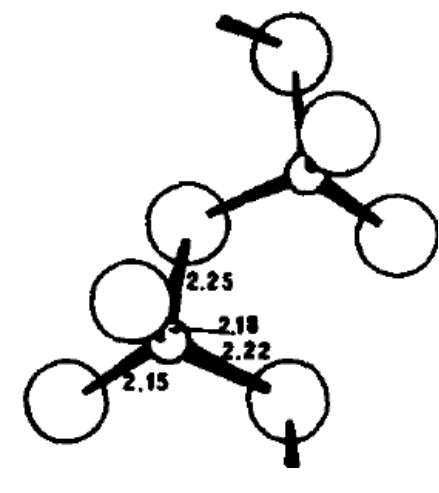

(b)

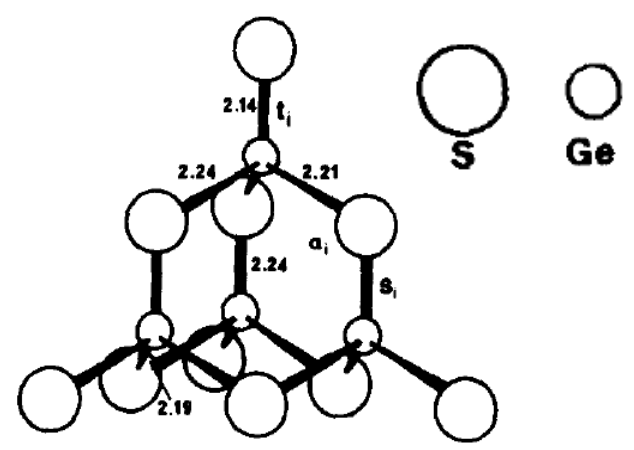

(c)

Figure 1.4: a) pyrothiogermanate; b) metathiogermanate; c) dithiogermanate structures

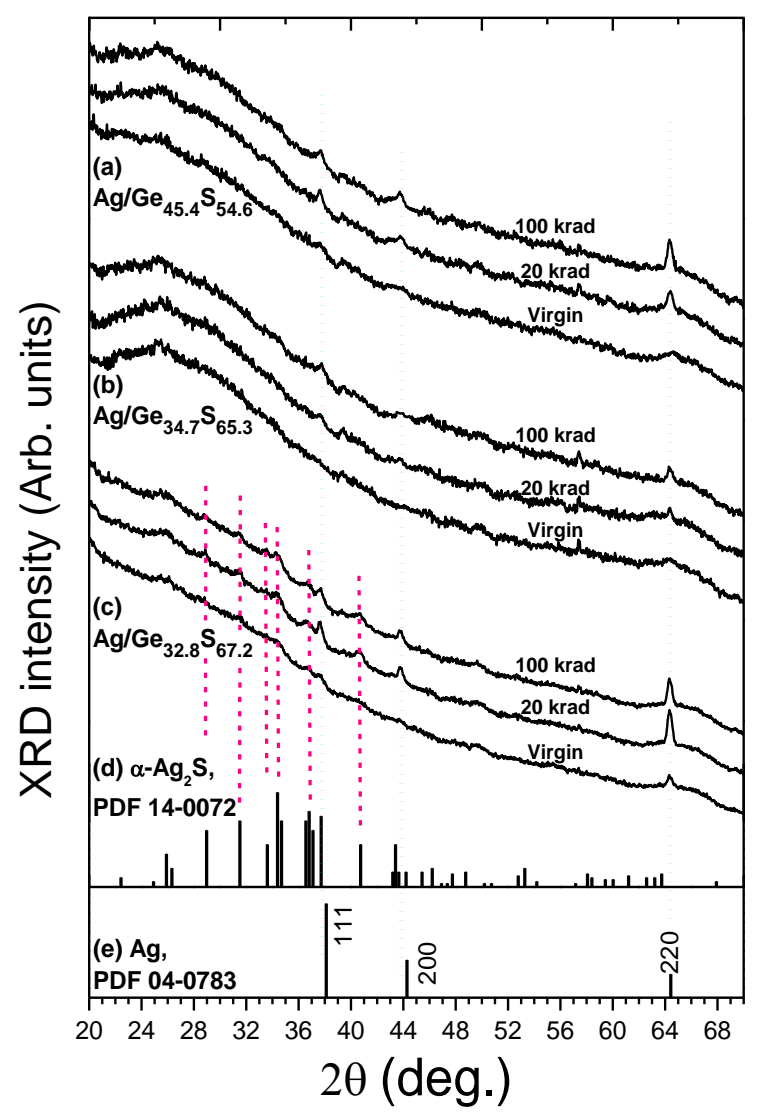

Figure 1.5: XRD data for the studied films at different radiation doses

The greatest structural change and the highest introduction of $\mathrm{Ag}$ was observed in germanium-rich films which is due to the formation of a layered structure in which both Ge and $\mathrm{S}$ are threefold coordinated through the formation of dative bonds. The possibility of a threefold coordination of both $\mathrm{Ge}$ and the chalcogen $\left(\mathrm{Se}\right.$ ) has been recently confirmed for liquid $\mathrm{GeSe}_{2}$ by 
first-principle molecular dynamic calculation [8]. The dative bonds are very weak and easily destroyable by radiation. This results in a high negative charge concentrated on the chalcogen atoms, which together with the channeled structure characteristic for this composition [10], is a big driving factor for $\mathrm{Ag}^{+}$diffusion into this glass. Since in this case there are no free valence electrons of S to react with Ag, we see on the XRD spectra Ag which is present in all studied cases because they all are high in Ge and show appearance of this type of structure on the Raman spectra. In addition, the demonstrated opening of the structure through reorganization of CS to ES structural units helps to the further introduction of Ag into the glassy matrix. It is for this reason that the conductivity increases much more for this particular glass compared to the cases with higher concentration of $\mathrm{S}$ as exampled on Fig. 1.6.

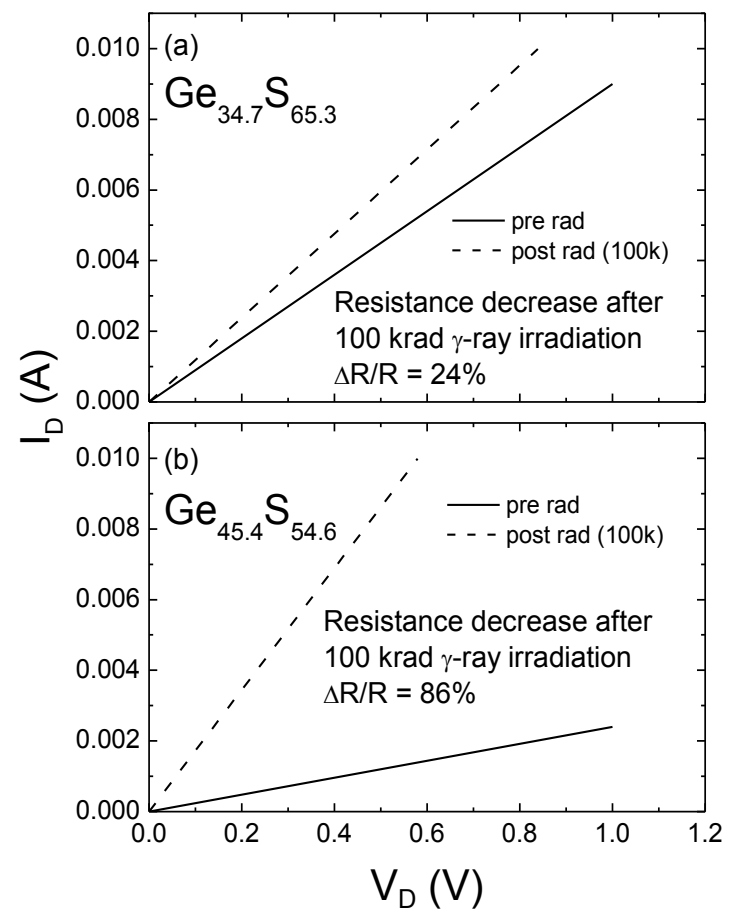

Figure 1.6: I-V curves of lateral radiation sensing structures before and after irradiation

Aforementioned structural changes can be measured by creating lateral devices, which have been fabricated using the same films that were used in the Raman, EDS and XRD study. Devices structure and fabrication are described in detail in a future section. The I-V characteristics show that after radiation the structural changes have been exhibited as change in resistance. The large reduction in resistance in the Ge-rich devices is supported by the structural reorganization and the increase in the $\mathrm{Ag}^{+}$diffusion in these films.

\subsection{Ge-Se Chalcogenide System - Influence of $\gamma$ Radiation}

The greatest structural change was observed in Ge-rich films similar to the case in the sulfur containing glasses. Even though, the size of selenium atoms is significantly greater than this of the sulfur atoms, this similar result is primarily attributed to the Ge-Ge bonds within the films, which are the weakest bonds. It was observed that with an increase in radiation dose, there was a decrease in the Ethane-like mode.

Other structural changes were observed in Ge-Se glasses from the Raman study. An increase in the Se-Se band at $263 \mathrm{~cm}^{-1}$ was observed in all compositions, where the strongest 
change occurred in the $\mathrm{Ge}_{40} \mathrm{Se}_{60}$ films and this change decreased in intensity in $\mathrm{Ge}_{30} \mathrm{Se}_{70}$ films with the smallest change being recorded in the $\mathrm{Ge}_{20} \mathrm{Se}_{80}$ films. There is also an increase in the ratio of CS/ETH structural units, which is also attributed to the breaking of the Ge-Ge bond. Additionally, since these experiments were performed in ambient environment, $\mathrm{Ge}-\mathrm{O}$ bonds can form, which are not detectable using Raman spectroscopy because of the low intensity.

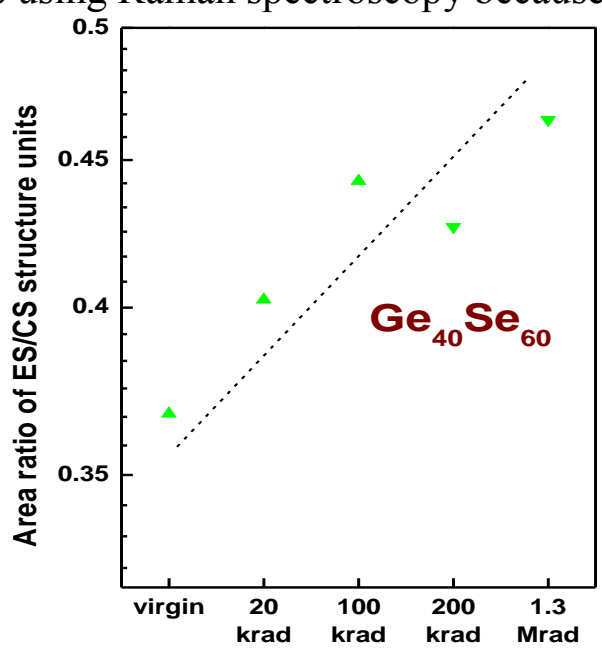

Figure 1.7: Dependence of the area ration ES/CS structural units for $\mathrm{Ge}_{40} \mathrm{Se}_{60}$ films at different irradiation doses

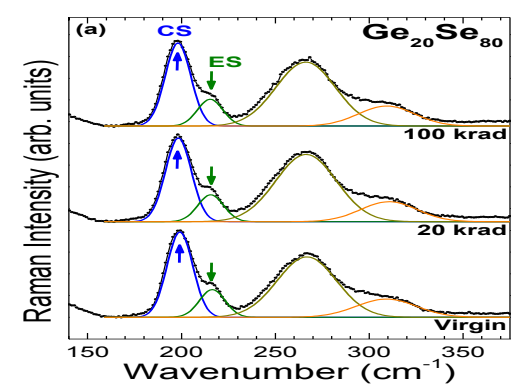

(a)

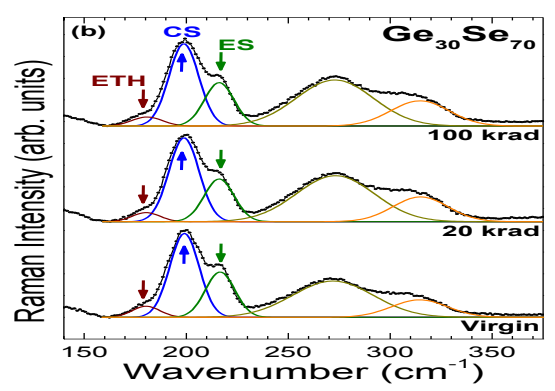

(b)

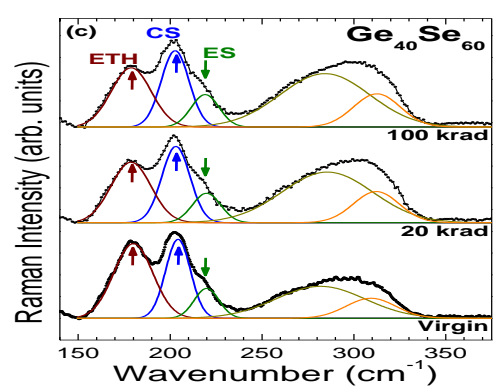

(c)

Figure 1.8: Fitted Raman spectra of virgin and radiated Ge-Se films for a) $\mathrm{Ge}_{20} \mathrm{Se}_{80}$; b) $\left.\mathrm{Ge}_{30} \mathrm{Se}_{70} ; \mathrm{c}\right) \mathrm{Ge}_{40} \mathrm{Se}_{60}$

A surface study of these films was performed using Atomic Force Microscopy (AFM). These studies showed that the roughness of the film changes due to irradiation. This confirms the formation of defects resulting in structural changes where the non-irradiated films are very smooth and increased radiation increases the roughness of the films.

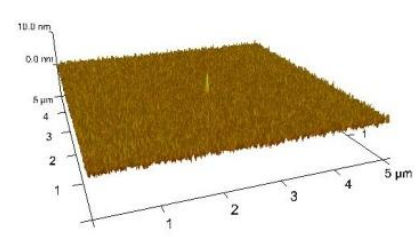

a) $\mathrm{R}_{\mathrm{q}}=0.59 \mathrm{~nm}$

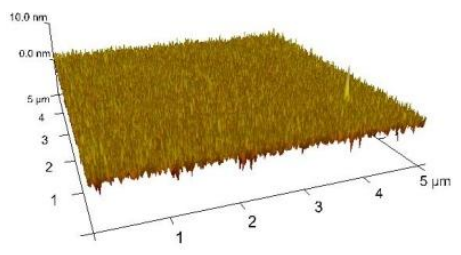

b) $\mathrm{R}_{\mathrm{q}}=0.90 \mathrm{~nm}$

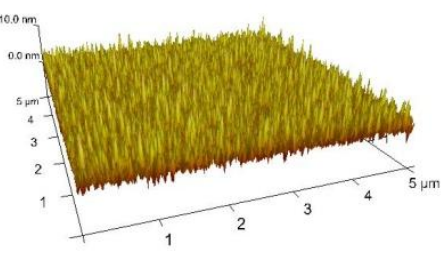

c) $\mathrm{R}_{\mathrm{q}}=1.34 \mathrm{~nm}$

Figure 1.9: AFM data from the surface studies of $\mathrm{Ge}_{20} \mathrm{Se}_{80}$ sample a) Virgin; b) $20 \mathrm{krad}$; c) $100 \mathrm{krad}$ 
Some specific films were also created to study the amount of silver introduced into the films due to radiation. These films were obtained by depositing a $50 \mathrm{~nm}$ continuous layer of silver on top of a bare chalcogenide thin film. After radiation, the excess silver film was dissolved in a $0.1 \mathrm{~mol} / \mathrm{L}$ solution of $\mathrm{Fe}\left(\mathrm{NO}_{3}\right)_{3}$ leaving behind only the amount of silver that was diffused into the film structure. Analysis of the EDS analysis shows that there is an increase of the silver concentration introduced into the system as a function of radiation dose. The structural analysis suggests that Ge-rich films would be the best acceptors of silver after the event of radiation because of the structural redistribution occurring in them which is characterized with increased molecular volume. However, selenium-rich films show that they are more sensitive towards accepting higher amount of Ag due to the high affinity between the Ag and the chalcogens. There is also obvious connection between the amount of the Ag diffused in these glasses and the molecular volume of the glasses. According to Feltz, increasing the germanium concentration up to $\mathrm{Ge}_{33} \mathrm{Se}_{67}$ glasses results in an increase in the molar volume with a maximum at this composition after which it decreases at a further increase in the germanium concentration [11] due to formation of a new type of structural building blocks ETH and distorted rock salt structures. The EDS result coincides with the development related to the molecular volume. The main take away from the EDS analysis in both types of films is the sharp rise in silver content after a threshold radiation dose has been achieved.
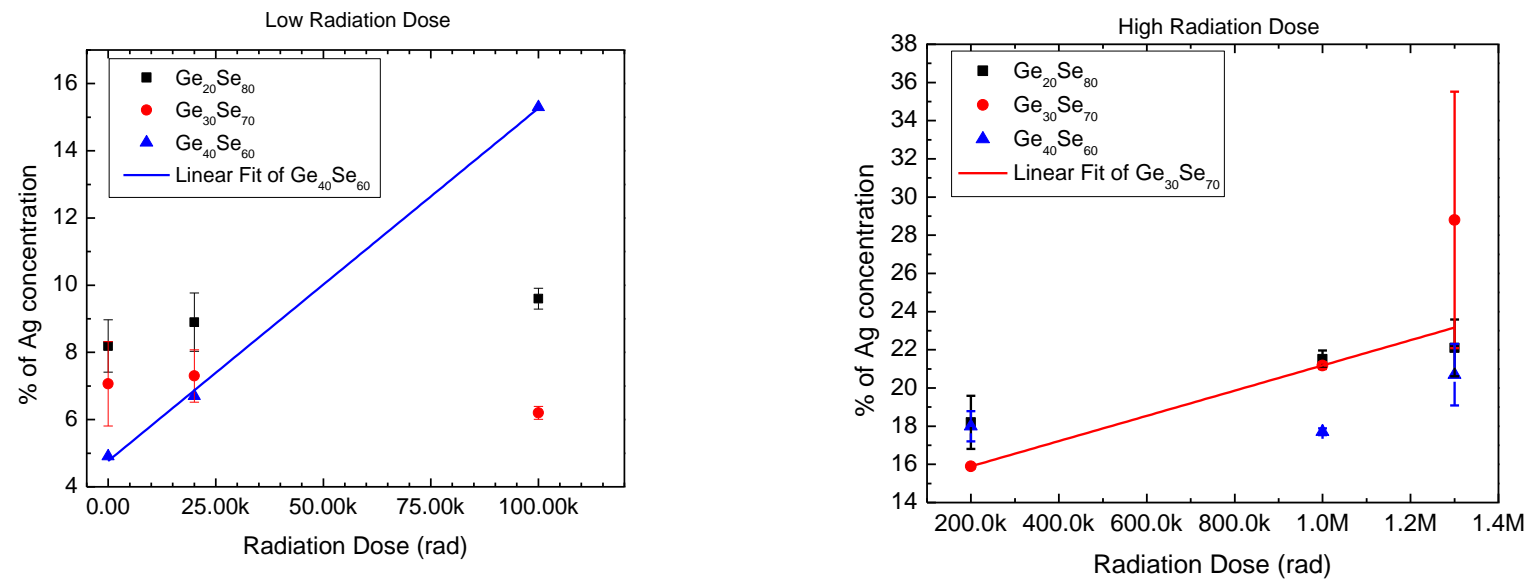

Figure 1.10: EDS analysis of $\mathrm{Ag} \%$ concentration at low and high radiation dose in Ge-Se films
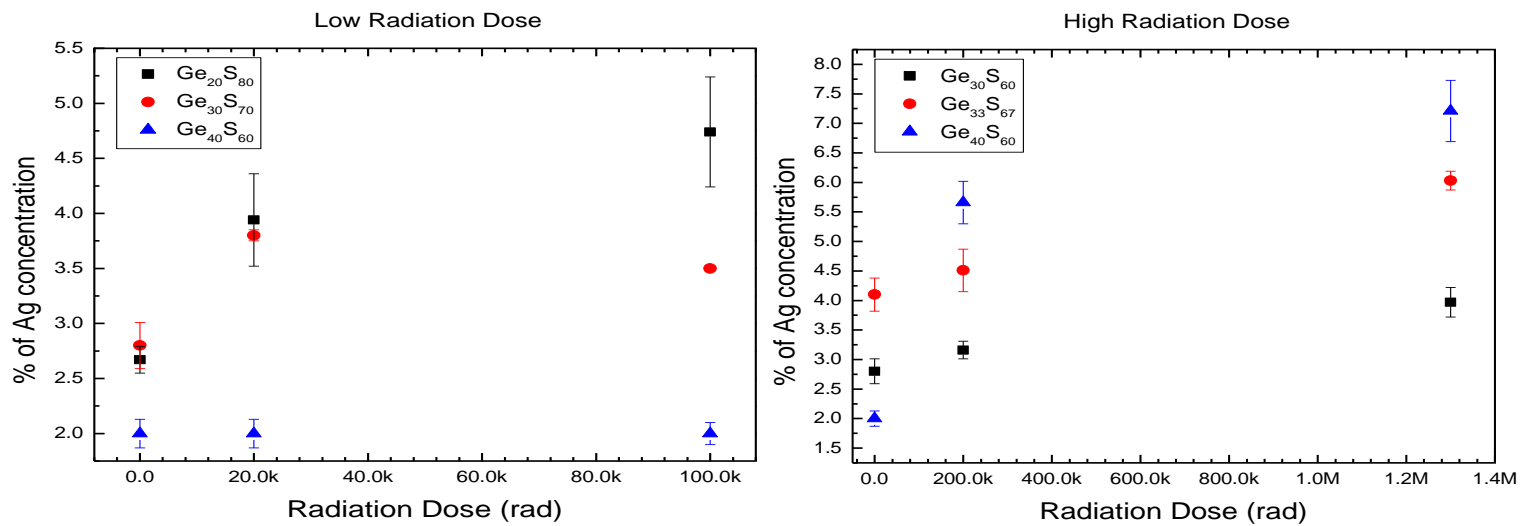

Figure 1.11: EDS analysis of $\mathrm{Ag} \%$ concentration at low and high radiation dose in Ge-S films 


\subsection{Influence of X-ray Radiation over the Chalcogenide Glasses and Ag Diffusion within the Structure}

The studies related to radiation of chalcogenide glasses with X-rays revealed that the radiation does not have a significant effect on the structure of chalcogen-rich films, while films containing 40 at.\% Ge undergo changes in their fine structure - Figure 1.12a. This affects the band gap, which incurs a slight increase after radiation - Figure 1.12b.
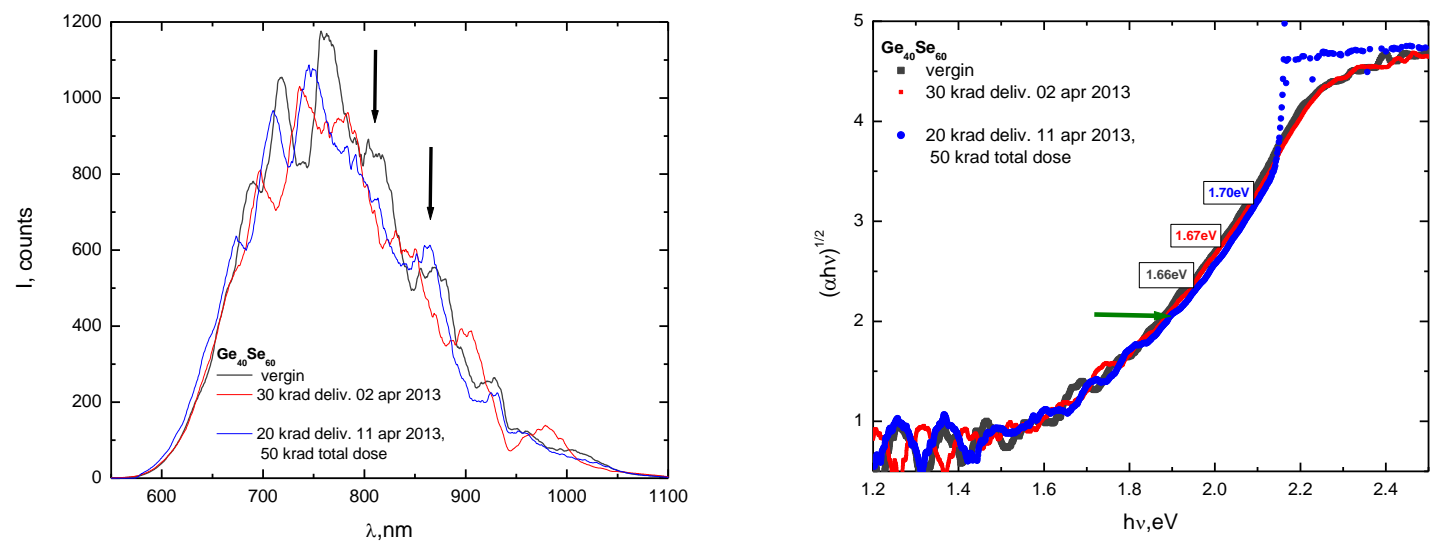

Figure 1.12: Influence of the X-ray radiation over $\mathrm{Ge}_{40} \mathrm{Se}_{60}$ thin films a) dependence of the absorption spectra upon the wavelength before and after X-ray radiation $b$ ) Influence of the $\mathrm{X}$-ray radiation over the band gap of the studied glasses

We assume that in the case of radiation with X-rays the effects are similar to films exposed to $\gamma$ rays. Hence for this reason we also observed structural changes only in the $\mathrm{Ge}_{40} \mathrm{Se}_{60}$ glasses.

Increased Ag diffusion as a function of the radiation dose has been proved for all film compositions.

XRD studies have been conducted in order to find out the molecular composition of the diffused products. Representative data in this aspect for the $\mathrm{Ge}_{30} \mathrm{Se}_{70}$ films are shown in Fig. 1.13 .
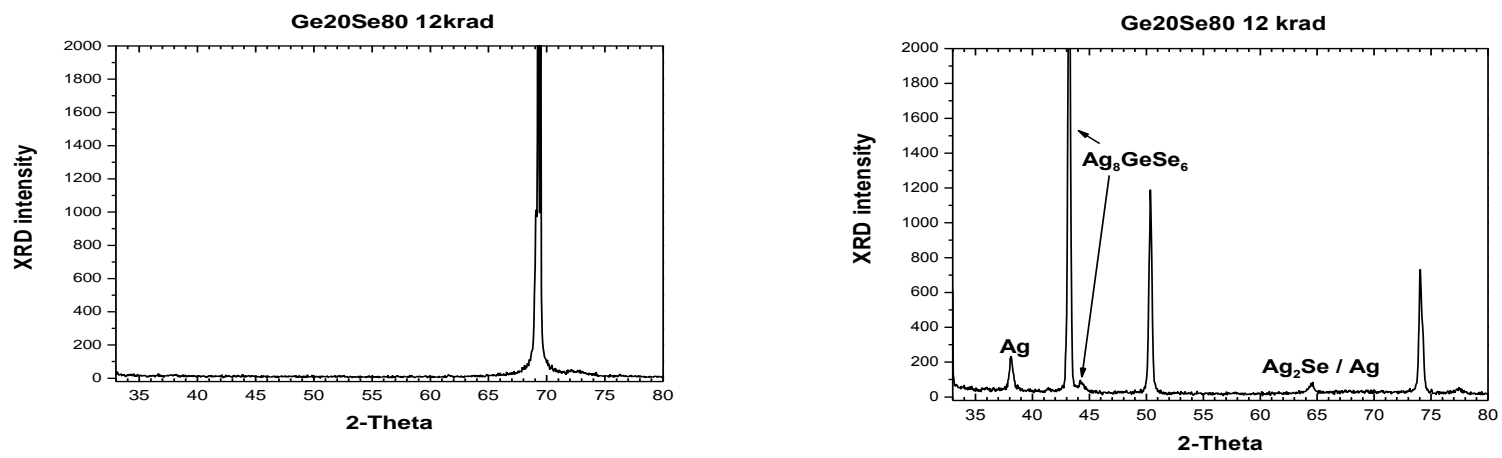

Figure 1.13: XRD study of $\mathrm{Ge}_{20} \mathrm{Se}_{80}$ glass before and after X-ray radiation showing the radiation products

The radiation induced reaction products show that there is a predominant formation of the ternary composition $\mathrm{Ag}_{8} \mathrm{GeSe}_{6}$. Additionally, part of the $\mathrm{Ag}$ has also formed the high 
temperature form - the cubic $\mathrm{Ag}_{2} \mathrm{Se}$ after reacting with the chalcogenide matrix. This implies that $\mathrm{Ag}_{2} \mathrm{Se}$ molecule is created within the established matrix and the surrounding structure limits its expansion and forces $\mathrm{Ag}_{2} \mathrm{Se}$ molecule to form in the closest packed structure, which is the cubic $\mathrm{Ag}_{2} \mathrm{Se}$. In this manner, the high temperature form is stable at room temperature. A radiation dose of $12 \mathrm{krad}$ plays a significant role in the growth of the peaks corresponding to the ternary $\mathrm{Ag}_{8} \mathrm{GeSe}_{6}$, which is attributed to the agglomeration of the new portions of diffused Ag. The growth of the Ag containing crystalline phases suppresses the appearance of the Si peaks generated from the substrate, which is evident from the before radiation XRD spectra. The introduction and growth of Ag containing products affects the overall structure of the hosting glass and it undergoes some distortion in order to accommodate the diffusion of Ag, which leads to a shift of the peak related to crystallization of the initial Ge-Se matrix.

\section{DEVICE DESIGN AND TESTING}

Multiple device structures were designed, which were created from similar films used in the film analysis described above. Since the device performance has a very strong relation to the structure, we accomplished many structural studies in parallel with the testing. Because they in essence confirm the preliminary materials studies, we do not show them in this report, but they are available in the quarterly reports.

\subsection{Lateral Device Structure with two Electrochemically Inert Electrodes}

These devices were created on a silicon wafer with a thermally oxidized insulating layer of $\mathrm{SiO}_{2}$. For performing electrical measurements, silver sources, which will diffuse laterally inbetween the measuring electrodes (tungsten or aluminum) were deposited using a circular shadow mask. The patterned device structure is shown in figure 2.1.

We developed a couple integrated circuit designs, which are suitable for measuring these devices. Since the measurement currents are very low, the measured devices are modeled as variable resistances. The curve is derived using the divider configuration showed below, which changes as the device resistance changes as simulated in the graph shown in Figure 2.2.

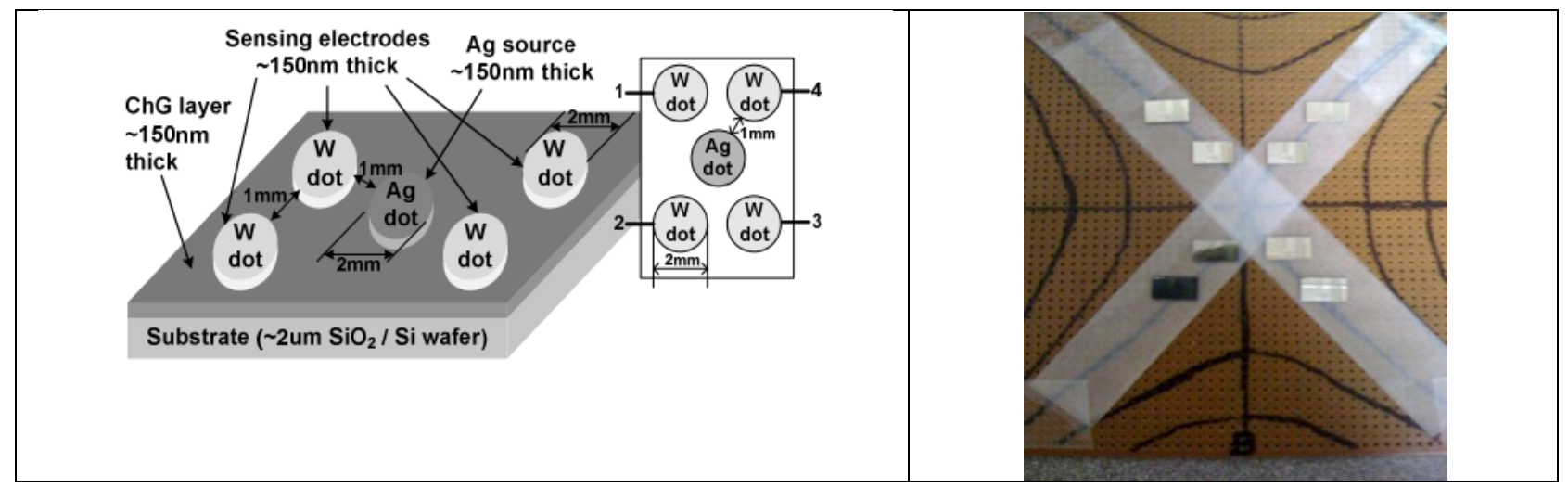

Figure 2.1: Lateral Device Structure patterned using a shadow mask and positioning of the devices on the highest radiation field distribution in the ${ }^{60} \mathrm{Co}$ camera 

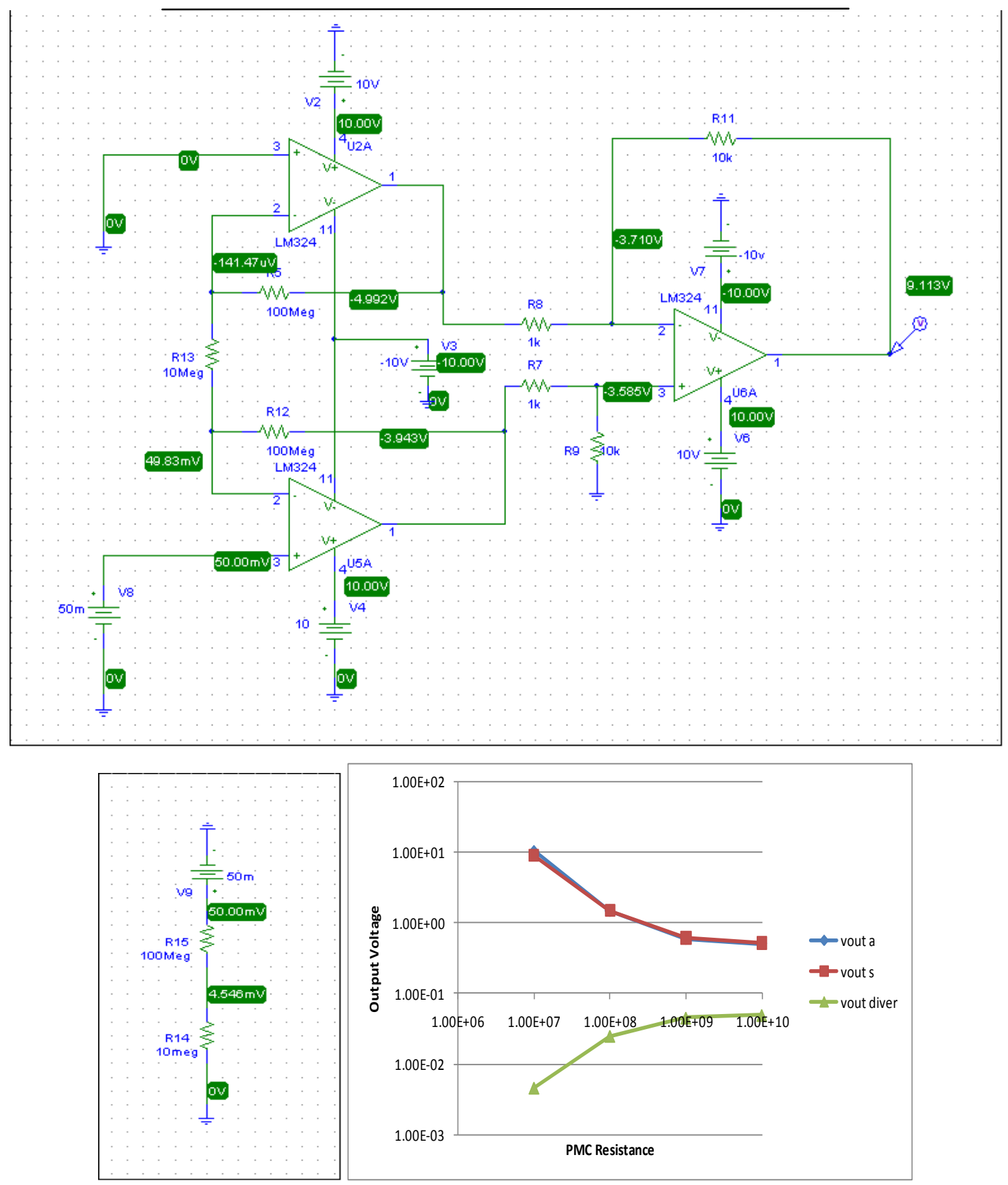

Figure 2.2: Testing schemes

Initially, these lateral devices were tested using visible light to check the functionality. The photon energies of visible light is significantly less than the energy of $\gamma$-radiation, but visible light can provide an insight towards the behavior under radiation. 


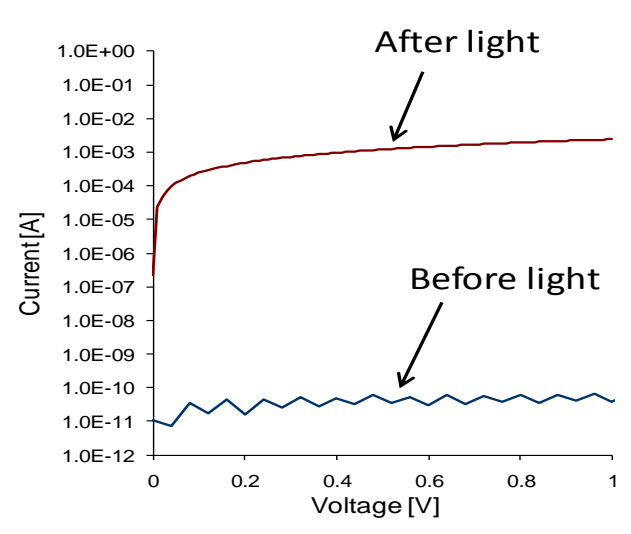

a)

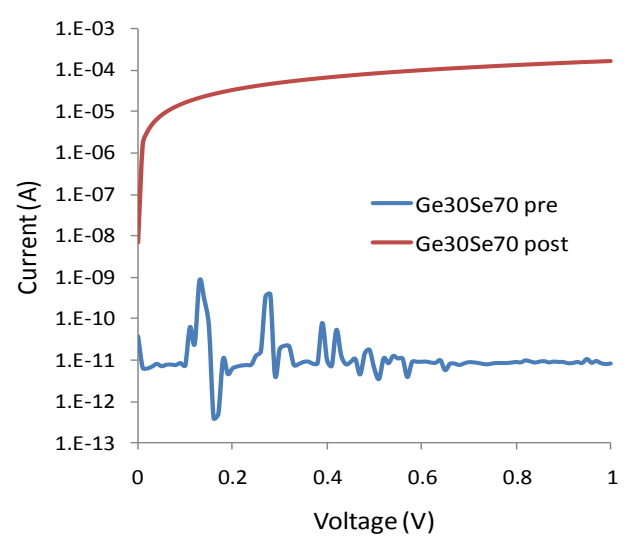

b)

Figure 2.3: I-V measurement before and after radiation a) $\mathrm{Ge}_{40} \mathrm{~S}_{60}$ device irradiated for 10,000 sec using visible light b) $\mathrm{Ge}_{30} \mathrm{Se}_{70}$ irradiated by $\gamma$-radiation with $100 \mathrm{krad}$

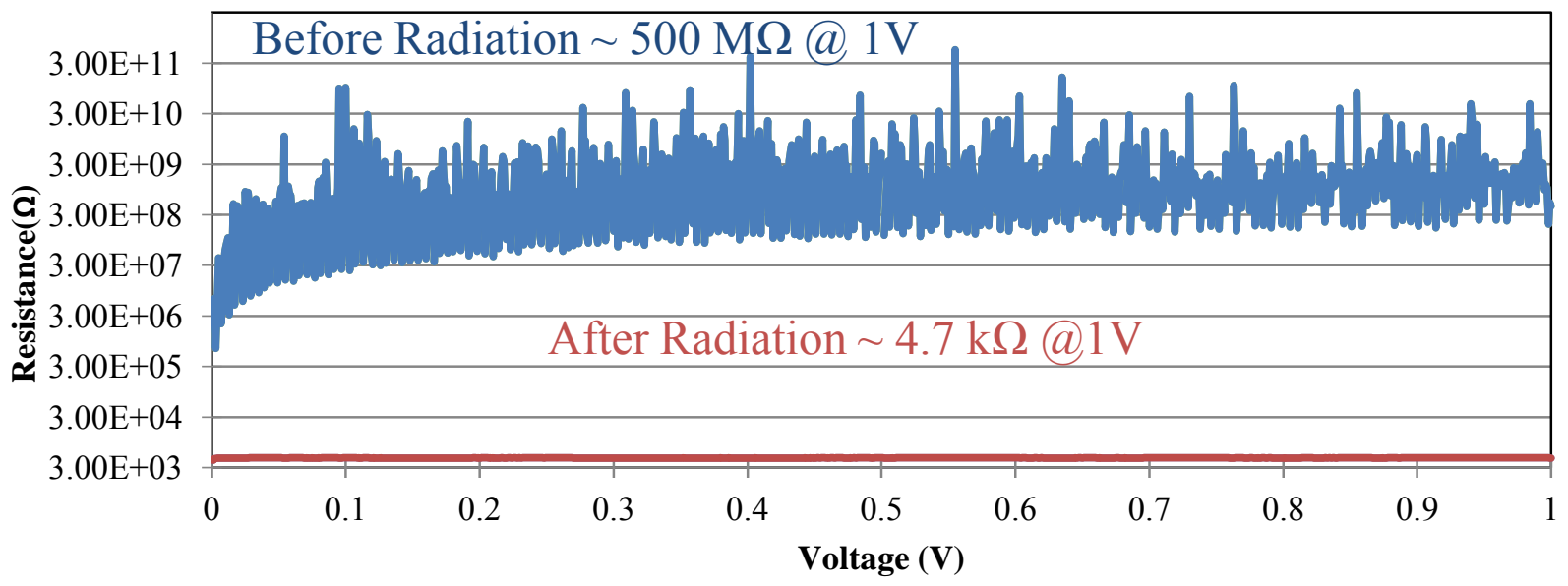

Figure 2.4: $\mathrm{Ge}_{20} \mathrm{Se}_{80}$ film before and after $4 \mathrm{hrs}$ of exposure to UV radiation

As shown in the electrical characterizations in Figure 2.3. and 2.4, these devices are highly reactive and show a significant change in the resistance.

There have been many studies which have correlated the effect of an applied voltage bias on the silver diffusion[12]. In our investigation, we studied the effect of an external bias on the performance of the radiation sensor as shown in figure 2.5. Application of a 1V bias (in device 1) between the two inert electrodes during the white light irradiation resulted in an increased current flow, suggesting that the bias is enhancing the movement of silver atoms and therefore it is difficult to determine whether the observed change in conductivity is a function of the bias or the radiation induced change. In the same experiment, device 2 was biased such that both the electrodes were at $0 \mathrm{~V}$ potential and the devices 3 and 4 were maintained at floating potentials. The main take away from this experiment is that devices fabricated on the same piece of silicon placed at a floating potential during the radiation exposure will behave in a similar manner, while application of any voltage bias will affect the device behavior. Due to this experiment, it was determined that all the gamma ray exposures has to be performed without a voltage bias for this type of lateral device structure. 

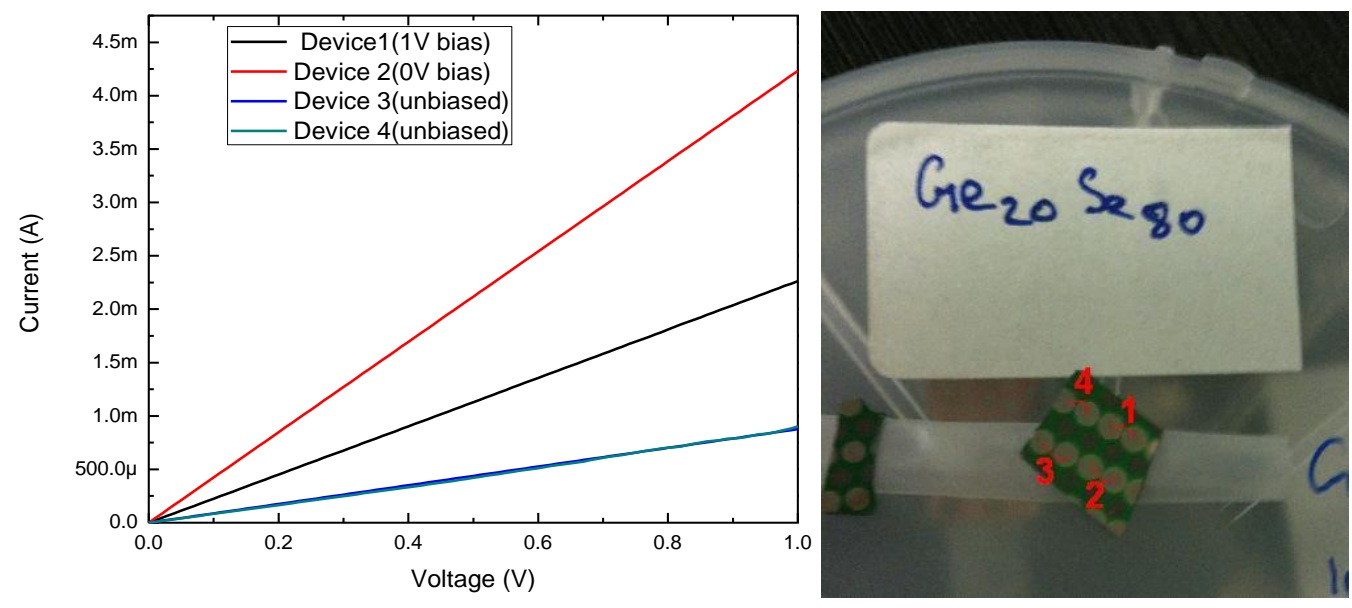

a)

b)

Figure 2.5: $\mathrm{Ge}_{20} \mathrm{Se}_{80}$ device tested after 10,000 sec of white light exposure a) I-V curves of all the devices b) Device image before irradiation

Further device testing is related to studying the dependence of the device performance upon the $\gamma$ radiation dose. It was performed for all studied compositions to demonstrate the compositional dependence of the devices performance as represented in figure 2.6. a-c. During the work on the project we performed detailed structural characterization of the materials related to the particular performance of the devices and analyzed it to relate performance to structure data are presented in the quarterly reports.

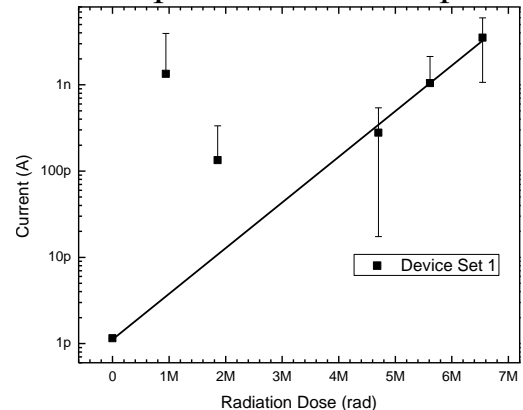

(a)

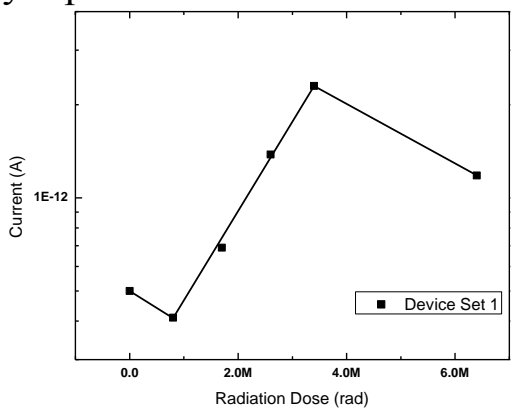

(b)

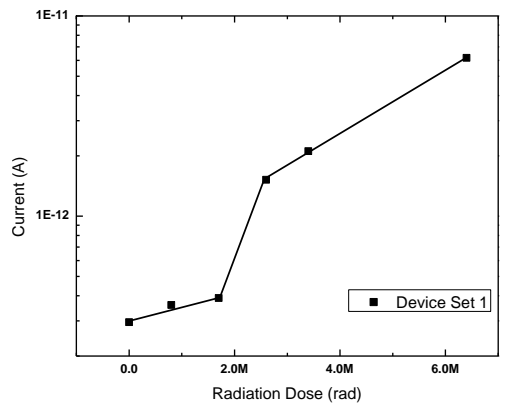

(c)

Figure 2.6: I-V curves of devices measured at various radiation doses $-\mathrm{a}) \mathrm{Ge}_{20} \mathrm{Se}_{80}$ b) $\left.\mathrm{Ge}_{30} \mathrm{Se}_{70} \mathrm{c}\right) \mathrm{Ge}_{40} \mathrm{Se}_{60}$

The sensor based on $\mathrm{Ge}_{20} \mathrm{Se}_{80}$ shows a constant increase in the current for low radiation doses, while the $\mathrm{Ge}_{40} \mathrm{Se}_{60}$ does not show a significant change in the current until higher radiation doses. Change observed in $\mathrm{Ge}_{30} \mathrm{Se}_{70}$ can be considered minimal due to the low sensitivity of the material. Structure of the films has a significant effect on the performance of the devices. We believe that in the case of $\mathrm{Ge}_{20} \mathrm{Se}_{80}$ films the prevailing role of the chalcogen, where formation of electron-hole pair is the predominant mechanism and contributes to the sensitivity. In the case of the $\mathrm{Ge}_{30} \mathrm{Se}_{70}$ glasses, the higher Ge concentration retards the sensitivity and contributes to the unstable devices performance. At higher Ge concentration - $\mathrm{Ge}_{40} \mathrm{Se}_{60}$ glasses the availability of Ge bonding and the strong tetrahedral bonding retards the sensitivity of the films at low radiation doses, but the availability of the weak Ge-Ge bonding contributes to bond breaking and the 
structural changes observed at the Raman studies which increases the devices sensitivity at higher radiation doses.

The opportunities for a real time radiation measurement were also explored using the 44 MeV LINAC at the Idaho Accelerator Center (IAC) at Idaho State University. We demonstrated that the radiation sensor proposed by us is capable for such measurements as shown on Figure 2.7, although we had to overcome many problems related to the very long line $(100 \mathrm{ft})$ between the probe and the measuring tool which at the low current that we were supposed to measure occurred as a very noisy signal.

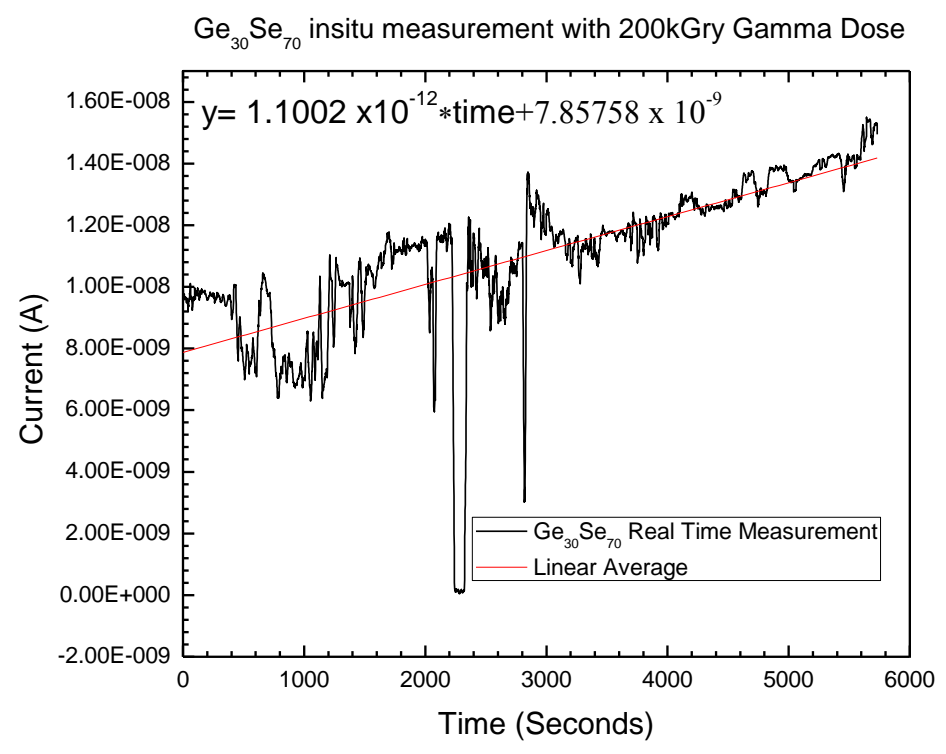

2.7. Real time radiation measurement at the $44 \mathrm{MeV}$ LINAC at the IAC

\subsection{Lateral Devices with Two Ag Electrodes}

These devices were prepared with application of a shadow mask as it was the previous case. The only difference was that both electrodes were made of Ag. This increases the sensitivity of the devices, but devices fabricated in this manner are at a disadvantage since these devices lose the reversibility capability that is possible with the other lateral devices. Once Ag diffuses away from the silver source, in the other lateral device, a voltage bias can be applied to recollect the diffused silver back towards the silver source; this procedure cannot be applied to this type of sensor design. The ideal operation of these devices is as a single use dosimeter due to the high sensitivity, inexpensive fabrication costs and high repeatability. The construction of thee devices is shown in Figure 2.8.

As in the previous cases, the performance of these devices was first checked by radiation with UV light after which they have been radiated with $\gamma$ rays. Among different members of the Ge-chalcogenide glass family, the $\mathrm{Ge}_{20} \mathrm{Se}_{80}$ composition has been chosen for studying this type of devices since the high amount of Selenium atoms aids in the introduction of high concentration of Ag through photodiffusion in this film [12,13]. 


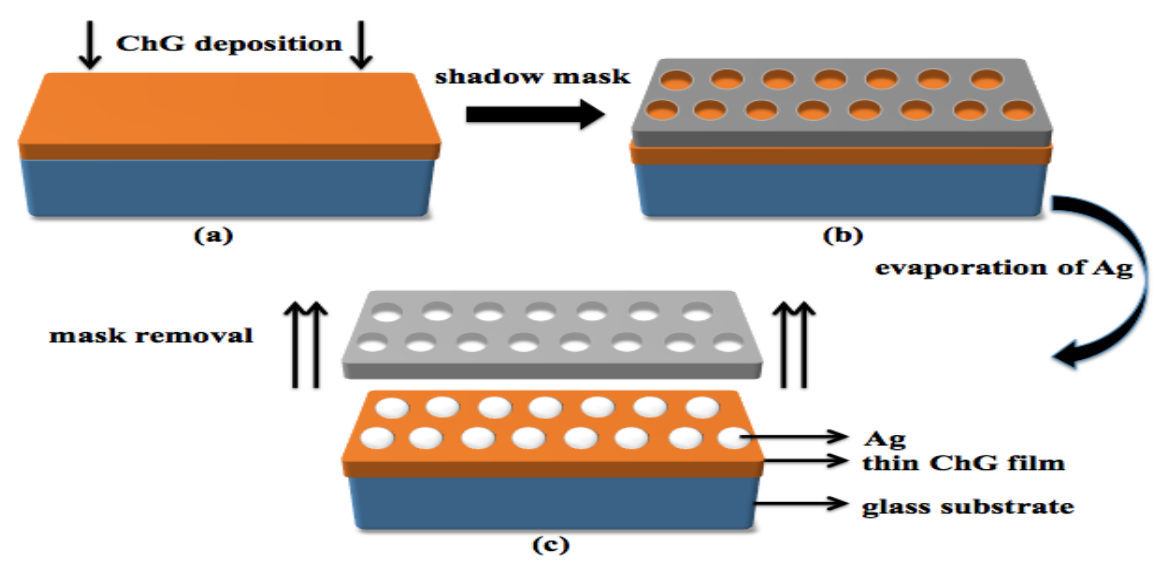

Figure 2.8. Lateral device with two Ag electrodes: Sensor fabrication process sequence

The irradiation protocol of these devices included first UV exposure as a simple and quick means for assessing resistance change due to photodissolution of the Ag electrodes as a function of exposure time. The UV light power density was $2.67 \mathrm{~mW} / \mathrm{cm}^{2}$ at a wavelength of $324 \mathrm{~nm}(1 \mathrm{hr}$ of UV exposure therefore corresponded to a total energy absorption density of $9.61 \mathrm{~J} / \mathrm{cm}^{2}$ ). For ${ }^{60} \mathrm{Co}$ gamma-ray exposure, the samples were placed in a Gammacell 220 irradiator with a dose rate of $10.5 \mathrm{rad}(\mathrm{GeSe}) / \mathrm{s}$. The samples were periodically removed from the Gammacell to measure the change in electrical resistance with respect to increasing dose levels. A maximum total ionizing dose (TID) of $1.5 \mathrm{Mrad}$ and 4.5 $\mathrm{Mrad}(\mathrm{GeSe})$ was reached in two different irradiation experiments. The samples were left floating (electrodes unconnected) during the exposures. Quasi-static DC electrical measurements were performed using an Agilent 4155B/C Semiconductor Parameter Analyzer (SPA) at room temperature. For these devices, the resistance between two adjacent $\mathrm{Ag}$ electrodes was monitored for $500 \mathrm{~s}$, at $10 \mathrm{mV}$ bias. The electrical measurements were carried out at such low-voltages in order to minimize Ag drift caused by internal electric fields during measurements. The diffusion of Ag in the chalcogenide glass was captured with an optical microscope - Figure 2.9.
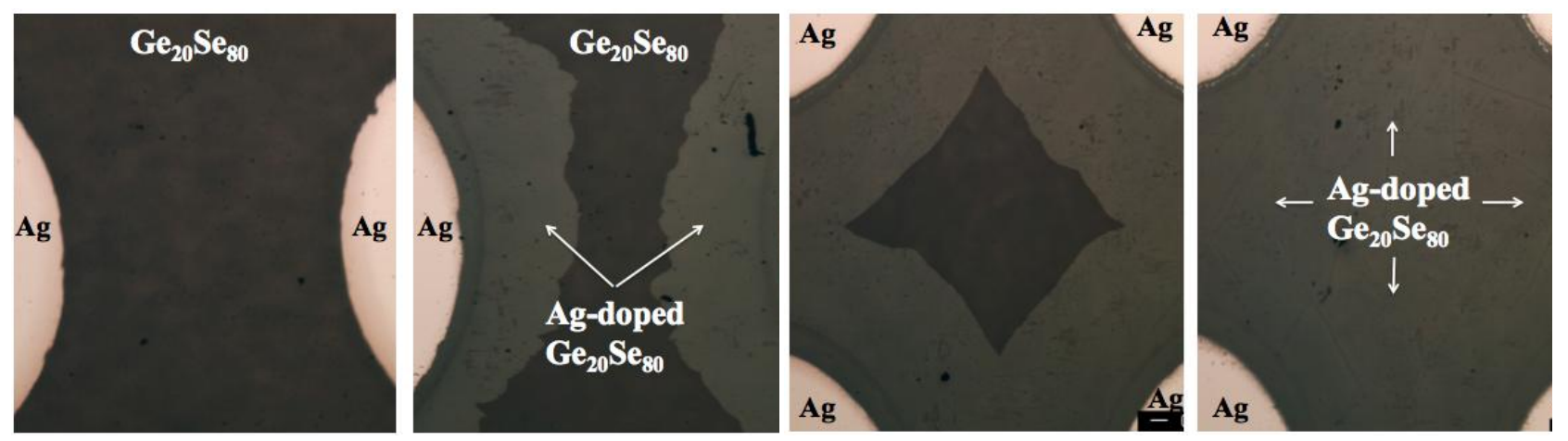

Figure 2.9. (a) Before (b) after $19.22 \mathrm{~J} / \mathrm{cm}^{2}$ (c) after $33.64 \mathrm{~J} / \mathrm{cm}^{2}$ (d) after $43.25 \mathrm{~J} / \mathrm{cm}^{2}$ of UV dose on device of $5 \mathrm{~nm}$ thick $\mathrm{Ge}_{20} \mathrm{Se}_{80}$.

Fig. 2.7.a shows two Ag electrodes on top of $5 \mathrm{~nm}$ thick $\mathrm{Ge}_{20} \mathrm{Se}_{80}$. These two electrodes are used as contact pads for electrical measurements as well as providing the Ag for incorporation into the ChG layer. Before UV exposure, the device exhibits a very high resistance state (OFF state). This OFF state resistance cannot be determined using the $4155 \mathrm{~B} / \mathrm{C} \mathrm{SPA}$, as the current is 
below the measurement limit of the instrument. After $19.22 \mathrm{~J} / \mathrm{cm}^{2}$ of UV exposure, as shown in Fig. $2.7 \mathrm{~b}$, Ag has diffused laterally by more than $0.5 \mathrm{~mm}$ between the two electrodes. At this stage, the device is still in the OFF-state, as there remains a region of undoped $\mathrm{ChG}$ between the measurement electrodes. As shown in Fig. $4 \mathrm{c}$, after $33.64 \mathrm{~J} / \mathrm{cm}^{2}$ of UV exposure, the ChG layer between the electrodes has been bridged by Ag-Ge-Se (doped) material. At this stage, the device is in a low-resistance 'ON-state'. The device resistance may be approximated by

$$
\begin{aligned}
R_{\text {OFF }} & =R_{\text {undoped }}+R_{\text {doped }} \approx R_{\text {undoped }} \\
R_{\text {ON }} & =R_{\text {undoped }} \| R_{\text {doped }} \\
& =\frac{R_{\text {undoped }} * R_{\text {doped }}}{R_{\text {undoped }}+R_{\text {doped }}} \approx R_{\text {doped }}
\end{aligned}
$$

where,

$$
\begin{aligned}
R_{\text {doped }} & =\rho_{\text {doped }} \frac{l}{w_{\text {doped }} * t} \\
R_{\text {undoped }} & =\rho_{\text {undoped }} \frac{l}{w_{\text {undoped }} * t}
\end{aligned}
$$

where $\rho_{\text {doped }}$ and $\rho_{\text {undoped }}$ is the resistivity of Ag-Ge-Se, ( $\rho$ depends on concentration of $\mathrm{Ag}$ in $\mathrm{Ge}-\mathrm{Se}$ ) and undoped $\mathrm{ChG}$ respectively, $l$ is the distance between two electrodes, $\mathrm{t}$ is the thickness of $\mathrm{ChG}$, and $\mathrm{w}$ is the width of doped or undoped region between the electrodes. Therefore, as the width of Ag-Ge-Se between two electrodes increases, the resistance is decreases.

The threshold dose $\left(\mathrm{D}_{\text {th }}\right)$ for radiation measurement can be defined as the point when the doped regions between two Ag electrodes are contacted, i.e. when a Ag-Ge-Se conduction pathway between the two electrodes is formed. As shown in Fig. 2.7c, although the ChG region between two electrodes has been Ag-doped, the longer region between 2 diagonal Ag electrodes has not been fully bridged. After $43.25 \mathrm{~J} / \mathrm{cm}^{2}$ of UV exposure, the undoped regions of ChG film have been completely doped with Ag, as shown in Fig $2.7 \mathrm{~d}$. The dose and resistance at this stage can be defined as saturation dose $\left(D_{\text {sat }}\right)$ saturation resistance $\left(R_{\text {sat }}\right)$, where $A g$ has completely saturated the $\mathrm{ChG}$ film. At this point, no further significant change in resistance with additional irradiation is evident.

Resistance as a function of exposure time was measured on a minimum of 20 device samples of $5 \mathrm{~nm}$ thick $\mathrm{Ge}_{20} \mathrm{Se}_{80}$ material and all devices showed similar response characteristics. Firstly, the influence of UV light was studied to establish the effects occurring. The resistance of each device was monitored for 500s and an average resistance was used in each case. Fig. 2.10.a) shows the resistance with respect to time and it is indicated that the state of the device has not been disturbed by bias measurements. After a dose of $43.25 \mathrm{~J} / \mathrm{cm}^{2}$, the resistance of the device was $2.5 \mathrm{k} \Omega\left(\mathrm{R}_{\text {sat }}\right)$ and does not show any significant change beyond this order of magnitude $\left(10^{3}\right)$ after $124.93 \mathrm{~J} / \mathrm{cm}^{2}$ of UV exposure. The OFF-state resistance is not plotted since it is beyond the instrument range. 

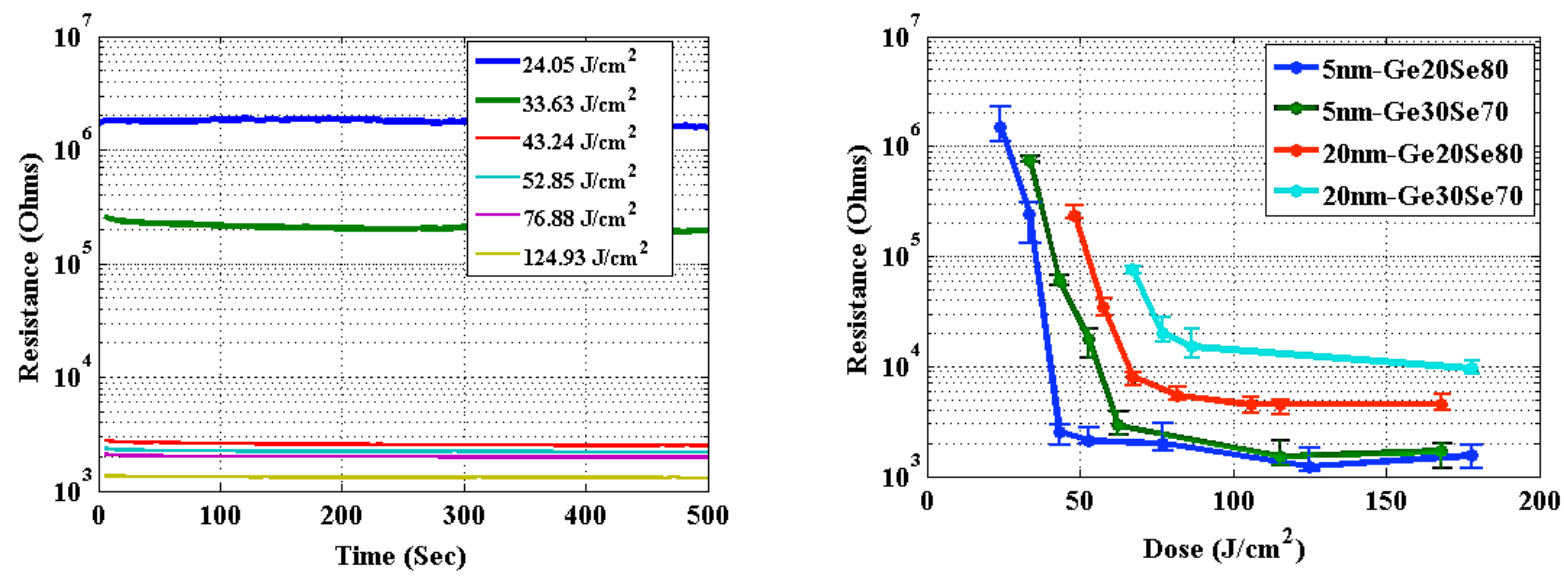

Figure 2.10. a) Resistance as a function of time of $5 \mathrm{~nm} \mathrm{Ge}_{20} \mathrm{Se}_{80}$ lateral devices at UV radiation; b) UV Dose Response Curve of lateral devices: Resistance as a function of total UV dose of $\mathrm{Ge}_{20} \mathrm{Se}_{80}$ and $\mathrm{Ge}_{30} \mathrm{Se}_{70}$ of $5 \mathrm{~nm}$ and $20 \mathrm{~nm}$ thicknesses.

It is evident that the composition of $\mathrm{ChG}$ can influence the $\mathrm{D}_{\text {th }}$ of the device. Also the difference, $\mathrm{D}_{\text {sat }}-\mathrm{D}_{\text {th }}$, is smaller in $\mathrm{Ge}_{20} \mathrm{Se}_{80}$ compared to $\mathrm{Ge}_{30} \mathrm{Se}_{70}$ devices, indicating that $\mathrm{Ge}_{20} \mathrm{Se}_{80}$ devices might have greater radiation sensitivity due to more rapid achievement of the threshold condition.

Gamma-ray Exposure Results

Gamma irradiations have also been performed on device samples and Fig. 2.11 shows the average resistance with respect to dose for $\mathrm{Ge}_{20} \mathrm{Se}_{80}$ devices with different thickness. The average threshold dose $\left(D_{\text {th }}\right)$ is $\sim 495 \mathrm{krad}\left(\mathrm{R}_{\mathrm{th}}=2.08 \times 10^{9} \Omega\right)$ and $\mathrm{D}_{\text {sat }}$ is $\sim 694 \mathrm{krad}$ $\left(\mathrm{R}_{\mathrm{sat}}=9.32 \times 10^{4} \Omega\right)$. Comparing Fig. 2.10 and 2.11., the devices show similar resistance change response to both UV and gamma radiation.

Although no significant difference has been observed in $\mathrm{D}_{\text {th }}$ of $5 \mathrm{~nm}$ and $10 \mathrm{~nm}$ devices, the $\mathrm{R}_{\text {sat }}$ of $10 \mathrm{~nm}$ is higher than $5 \mathrm{~nm}$ device, suggesting higher resistivity in $10 \mathrm{~nm}$ device (for constant $\mathrm{Ag}$ electrode thicknesses). Also the response time $\left(\mathrm{D}_{\text {sat }}-\mathrm{D}_{\text {th }}\right)$ is lower in $5 \mathrm{~nm}$ devices (105 krad), compared to $10 \mathrm{~nm}$ device (199 $\mathrm{krad})$, therefore indicating that Ag diffusion riches saturation faster in thinner films.
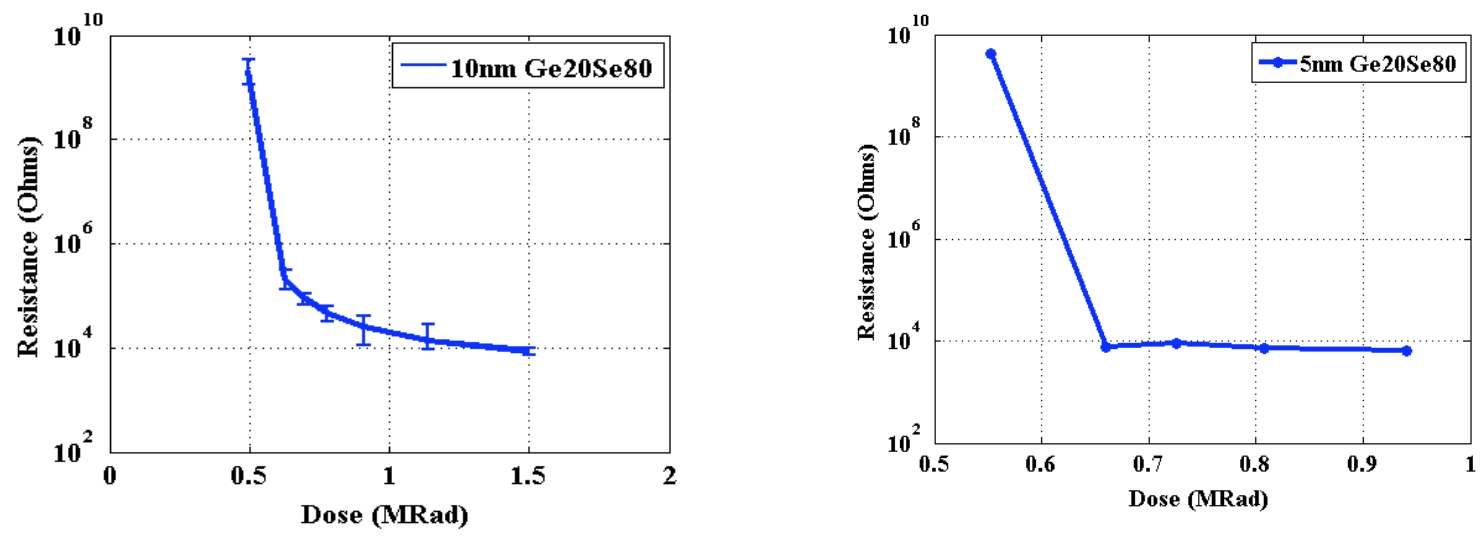

Figure 2.11.a)Resistance as a function of gamma TID of $10 \mathrm{~nm} \mathrm{Ge}_{20} \mathrm{Se}_{80}$; b) same for $5 \mathrm{~nm}$ devices 
After the devices reach a saturation dose of $660 \mathrm{krad}$, the $5 \mathrm{~nm} \mathrm{Ge}{ }_{20} \mathrm{Se}_{80}$ devices were further exposed to a TID of $940 \mathrm{krad}$ and no significant change in resistance was observed.

\subsection{Layered Devices}

The following device structure also known as layered device was created to take advantage of the dynamic and the measured static changes in the Ge-rich chalcogenide. This can be achieved by having a source of silver atoms within proximity towards the measuring electrodes.

This new type of sensor design uses a similar silicon substrate with an oxidized insulator as the lateral devices. Without breaking vacuum, an additional $100 \mathrm{~nm}$ of $\mathrm{Ge}_{40} \mathrm{Se}_{60}$ insulating chalcogenide glass $(\mathrm{ChG})$ layer was evaporated, followed by a $50 \mathrm{~nm}$ of silver. The purpose of the insulating layer is to ensure that the silver layer is not a continuous layer creating two series connected opposite polarized Programmable Metallization Cell (PMC) devices. This issue is partially resolved because during the evaporation of silver, the silver atoms will partially diffuse into the insulating chalcogenide matrix, which was observed in the EDS study of the bare films topped with silver. In this manner, only part of the theoretical $50 \mathrm{~nm}$ thickness will still be present above the $100 \mathrm{~nm}$ of chalcogenide film. The final deposition step, while still under vacuum, was the evaporation of a $300 \mathrm{~nm} \mathrm{Ge}{ }_{40} \mathrm{Se}_{60}$ chalcogenide thin film where the radiation will have an initial interaction with the chalcogenide film. This entire film stack was fabricated under vacuum to prevent the introduction of contaminants between each layer. After the film stack fabrication, metal electrodes were deposited on top and the final device cross section is shown below.

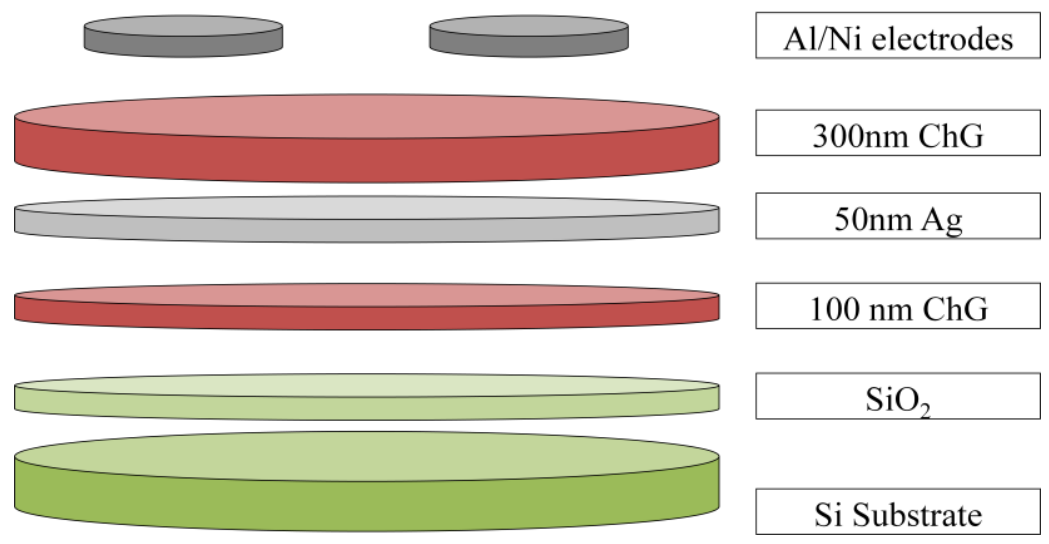

Figure 2.12: Layered Device Cross Section

Another manner to avoid the creating two PMC devices was to use low measuring voltages. Several experiments were performed to determine the maximum allowed voltage without affecting the device performance. The device was initially measured using an impedance meter with a $\mathrm{V}_{\text {ac }}$ of $10 \mathrm{mV}$ followed by a current vs. voltage (I-V) sweep and this process was repeated. The secondary impedance spectra and I-V was compared to the first sweep measurements. It was determined that a voltage sweep from $0 \mathrm{~V}$ to $200 \mathrm{mV}$ was a suitable one, which does not affect the device behavior. In this manner, the effect of the creating two PMC devices has been avoided by this measurement restriction. Data about the performance of $\mathrm{Ge}_{20} \mathrm{Se}_{80}$ based devices as a function of the dose are presented in Figure 2.13. 


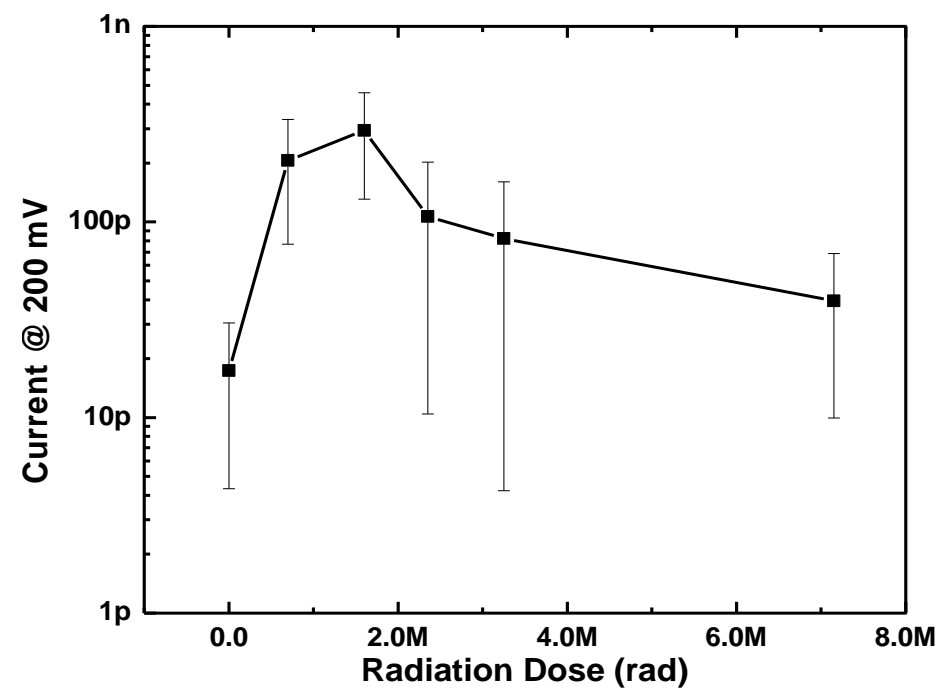

Figure 2.13: Current vs. Radiation dose measurements for $\mathrm{Ge}_{40} \mathrm{Se}_{60}$ devices

The device measurements at low radiation doses, work in cohesion with the previous type of devices and the Raman as well as the XRD results, where we observe a rise in the current due to the increased amount of silver diffusion along with no strong structural changes. However, the question arises to the reasoning behind the decline of current with increased radiation dose above 2Mrad. Although there is increased amount of Ag diffusing in the material, the conductivity diminishes for which oxidation could be a reason, since the radiation and the testing have been performed in the laboratory environment.

We suggest that at low radiation doses Ag diffuses in the glass matrix and forms the diffusion products, as discussed in the film characterization section. However, with increasing amount of Ag entering the films, as a result of its reaction with the chalcogen atoms there are a number of Ge atoms left behind. They have unsaturated bonds, which are very reactive and could interact with the environmental oxygen. We did not detected the appearance of Ge-O bonds since they have a very low activity on the Raman spectra and may be the clusters that germanium oxide forms are so small that they are undetectable using XRD.

There is one other method to check whether the presence of oxygen plays a role on the device performance. This technique is to measure the device in two different environments, in the ambient at room temperature and in a vacuum environment and compare the two results. The device under vacuum was placed inside a lakeshore cryostat and pumped down to $1 \times 10^{-5} \mathrm{mbar}$. For this experiment a $1.5 \mathrm{~W} / \mathrm{cm}^{2}$ Firefly UV lamp was used to simulate the effect of gamma radiation. A continuous $100 \mathrm{mV}$ bias was applied using a Keithley picoammeter, and the current was simultaneously measured as a function of time. A $100 \mathrm{mV}$ bias was chosen to ensure that the voltage potential would not play a role in the measurement of the device. 


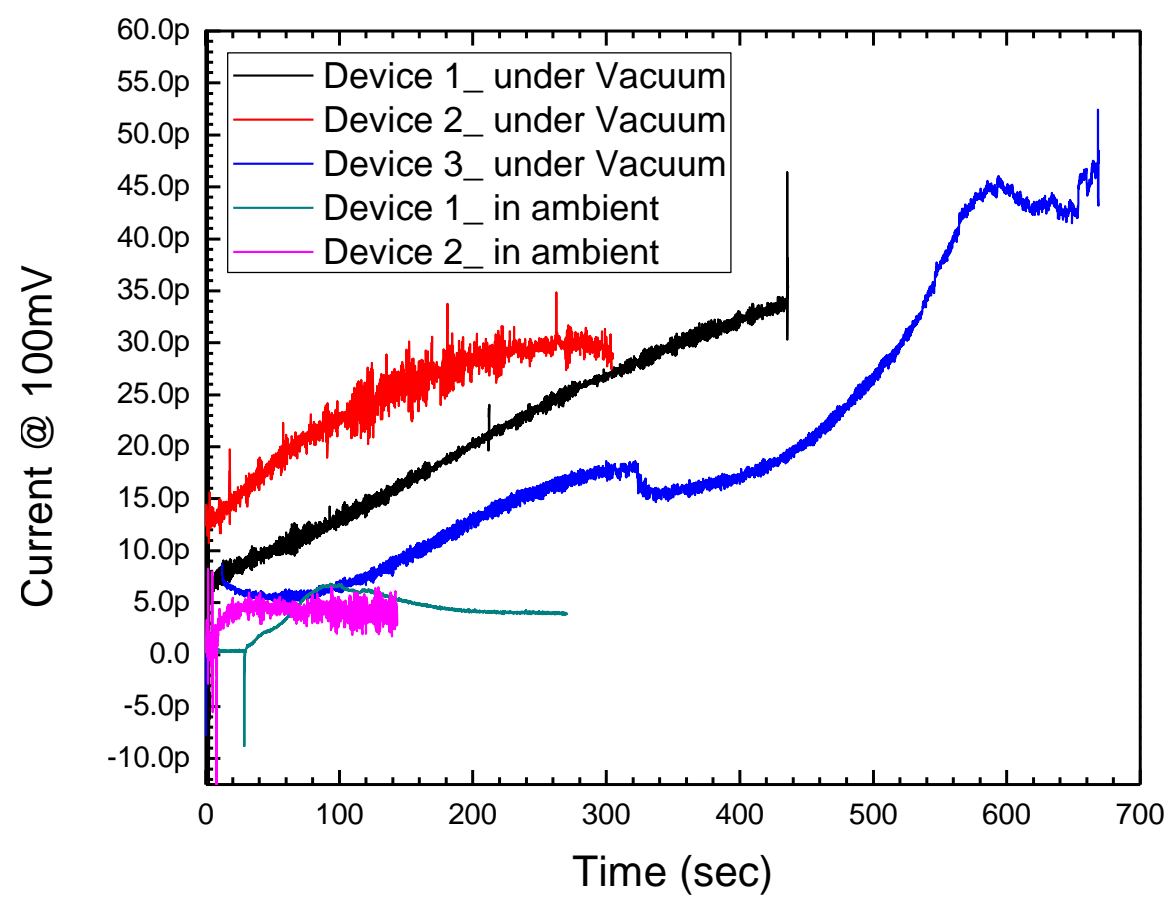

Figure 2.14: In situ measurement under vacuum and in ambient

The result of this experiment shows that at ambient environment, the current initially begins to rise but then after a sufficient time, the current begins to level off or decrease which is the case for Device 1 in ambient. On the other hand, the devices under vacuum show a continuous increase in the current with increasing the radiation time. This result shows that the presence of oxygen immensely affects the device performance.

The layered devices have also been measured using an impedance meter in addition to measuring the I-V curves after the discrete radiation doses as shown in Figure 2.15. In addition to the experimentally measured data, these devices have also been modeled using an equivalent $\mathrm{RC}$ model derived from the impedance measurements.

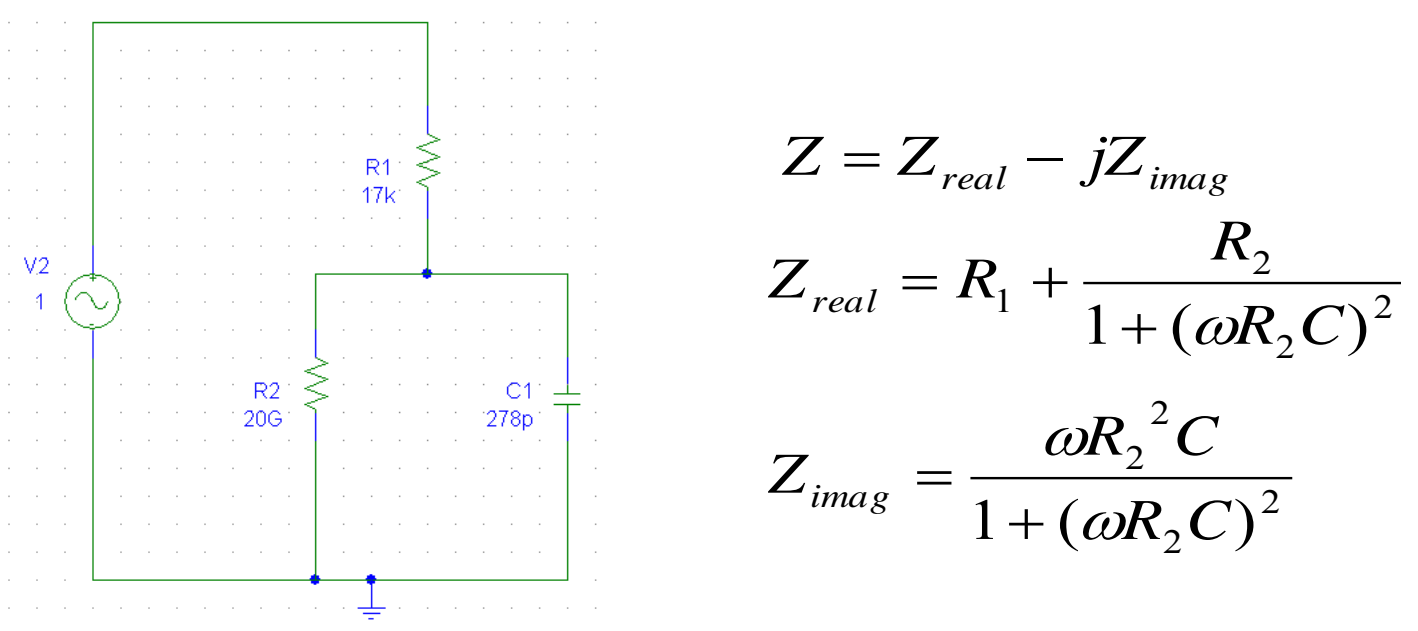

Figure 2.15: RC equivalent circuit and equations governing the analytical model 
Along with the analytical modeling of the device, this device was also modeled using SILVACO Atlas finite element two-dimensional device simulator using the same thickness as the deposited films.

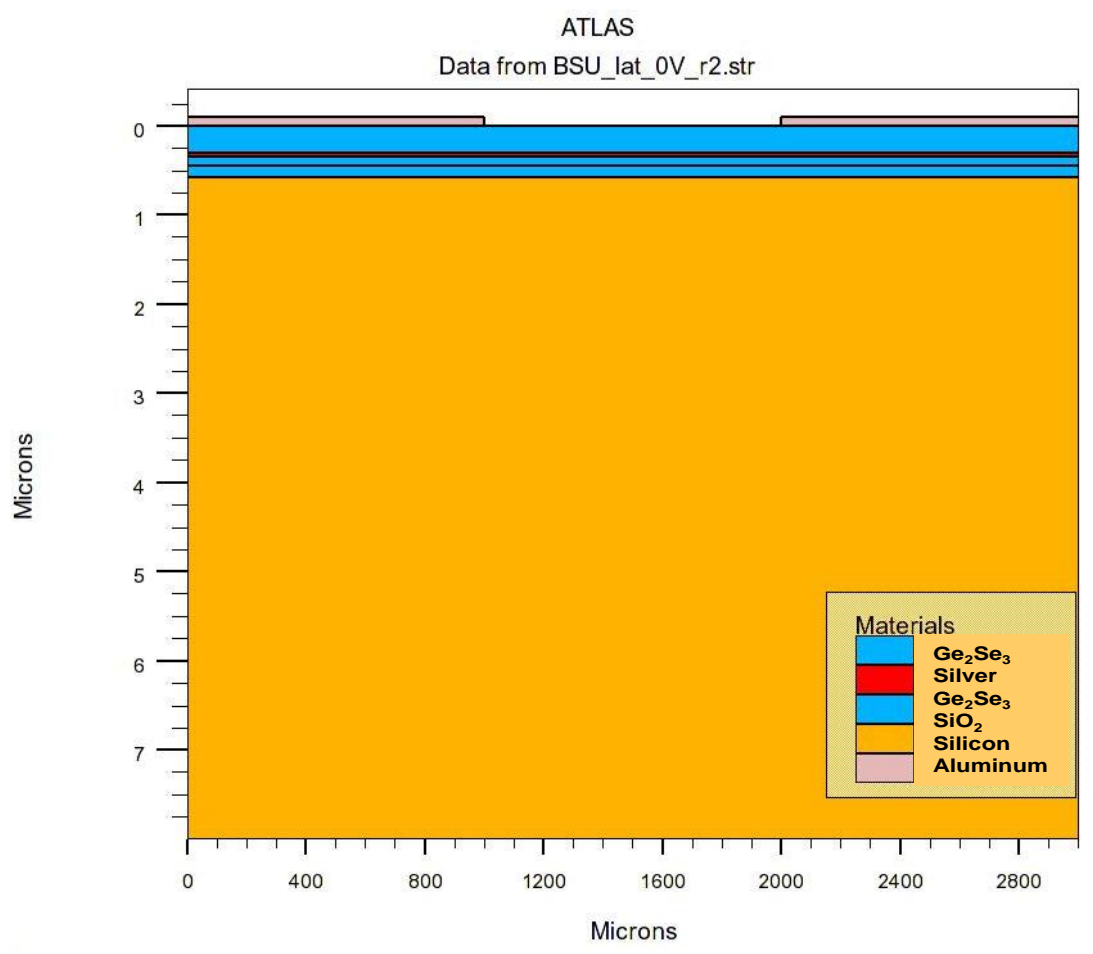

Figure 2.16: SILVACO Modeling of Layered Device

To compare the accuracy of the analytical and the simulation models with the actual results, the measured magnitude and phase of the impedance was compared to the calculated magnitude and phase of the impedance. The results show a good agreement with the modeling and the experimental data as shown in figure 2.16.

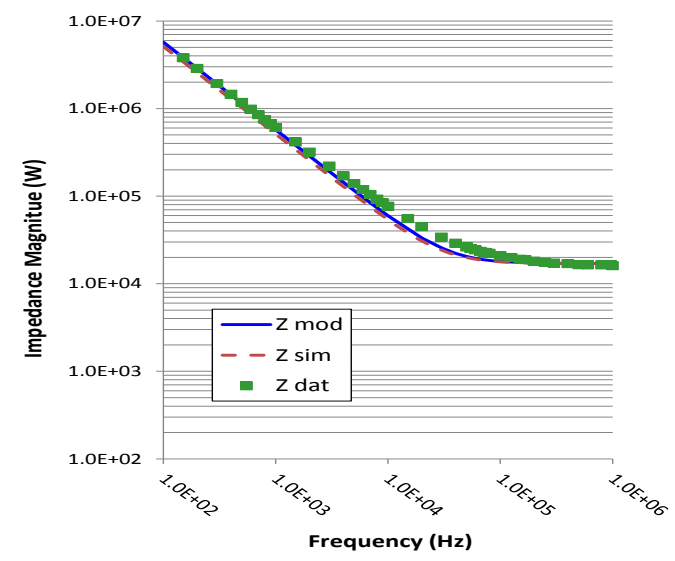

(a)

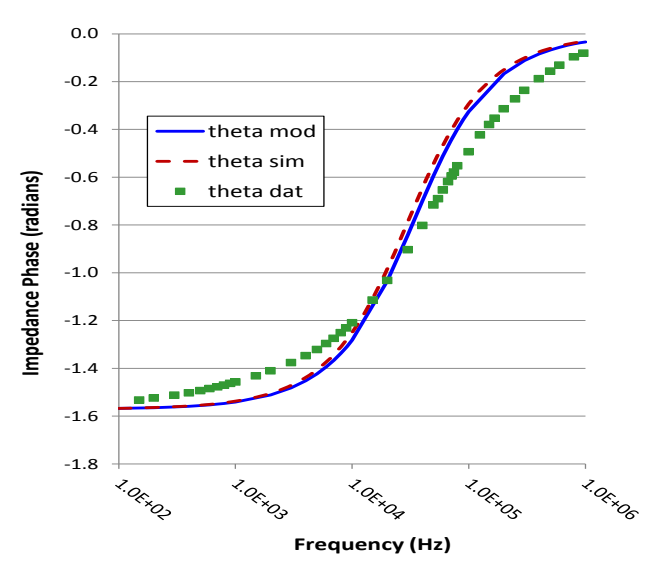

(b)

Figure 2.17: Experimental data (dat), SILVACO simulation (sim), analytical model (mod); a) Magnitude of impedance vs. Frequency; b) Phase of impedance vs. Frequency 
The final goal for the creation of these radiation sensors is to create a device that could be embedded into current semiconductor fabricated circuits. For this reason, simulations were performed to determine an ideal lateral device design and process flow was developed which is described in the following section.

\subsection{Simulations Based Device Design of Electrical Field Optimized CMOS-Compatible Devices}

The effect of silver migration in chalcogenide glasses and specifically germanium containing glasses under the influence of electric field has been thoroughly studied [12]. The electric field also affects the Ag photodoping [13]. From these studies, it turns out that the application of an E-Field during the testing of the radiation sensing devices can influence the diffusion parameters and introduce E-Field related effects, which can cloud the results from radiation-induced effects. Therefore, using COMSOL multiphysics software it is possible to calculate the electric fields for an applied voltage and vary the device dimensions to manipulate the E-field to achieve an ideal geometry that will allow for the measurement of the radiation effects without the influence of electric field. Materials data used for simulation were carefully selected to ensure the closest possible similarity to the fabricated thin films. A large square was created to represent the large area for the thin chalcogenide film and the composition of $\mathrm{Ge}_{20} \mathrm{Se}_{80}$ was used in the simulation with a relative permittivity of 6.98 [14]. The metal pads were simulated using material data that was inbuilt into the software, which used aluminum for the measuring electrodes and the data pertaining to silver for the silver source. During the sensing procedure, a constant 1-volt bias is applied to one of the aluminum electrode and the other aluminum electrode is biased at 0 volt. Application of a potential bias causes an E-field, which has been simulated for each of the geometries. Same method has been applied for simulating the electrical energy that is imparted into the film due to the voltage bias. The electrical energy density provides evidence of the amount of energy transferred to an ion in certain location, measured in Joules $/ \mathrm{m}^{3}$.

The sizes of the shapes have been scaled to make them proportional to the distance between the pads. The circle pad sizes used in the simulation were $2 \mathrm{~mm}$ in diameter with $1 \mathrm{~mm}$ spacing. The dimensions of all shapes in certain simulations were the same to prevent any anomalies due to distorted shapes. The simulations also contained a parameter that accounts for the $100 \mathrm{~nm}$ film thicknesses, which is referred in COMSOL as "Out of Plane Thickness".

Another important aspect for consideration, included in the software, is the amount of energy imparted into a given space or medium as a direct result of the voltage bias. This is also known as the energy density due to electric and magnetic field, of which the electric energy density is proportional to the permeability and the E-field as shown in the following equation:

$$
\eta_{E}=\frac{\text { energy }}{\text { volume }}=\frac{1}{2} \varepsilon E^{2}
$$

Here $\eta_{E}$ is the energy density due to electric field, $\varepsilon$ the dielectric constant of the material. It is critical to measure this energy since electron-hole pair (EHP) could also be generated as a direct result of the E-field. Complete energy equation also considers the role the magnetic fields plays in the system, with $U$ being the total energy, B the magnetic field, $H$ the magnetizing field, $\mathrm{D}$ the electric displacement field, and $\mu$ magnetic permeability. Then the total energy could be calculated as follows:

$$
\begin{array}{r}
U=\frac{1}{2}\left(\varepsilon E^{2}+\frac{B^{2}}{\mu}\right) \\
U=\frac{1}{2}(E \cdot D+\underset{H}{H} \cdot B)
\end{array}
$$


The total energy has been contemplated in the simulations, which guarantee that all external field factors that additionally could affect Ag diffusion are considered. When discussing Ag doping in chalcogenide glasses due to radiation induced effects, it is important to consider that an intrinsic E-field is also generated. It occurs because the thermally generated electrons in the doped region move towards the non-doped part, generating a field at the interface, which is limited by the equilibrium conditions, characteristic for diffusion and drift of the charge carriers. It contributes to the changes of the electrical conductivity of the film as a subset of the change in conductivity during radiation, and is accompanying the Ag diffusion. After cession of the radiation, the relaxation of the radiation-induced electron is associated with ion relaxation of the diffused Ag. This intrinsic field dominates at low external voltage that is used to measure the conductivity in the device. Results from this simulation are shown in Figure 2.18.

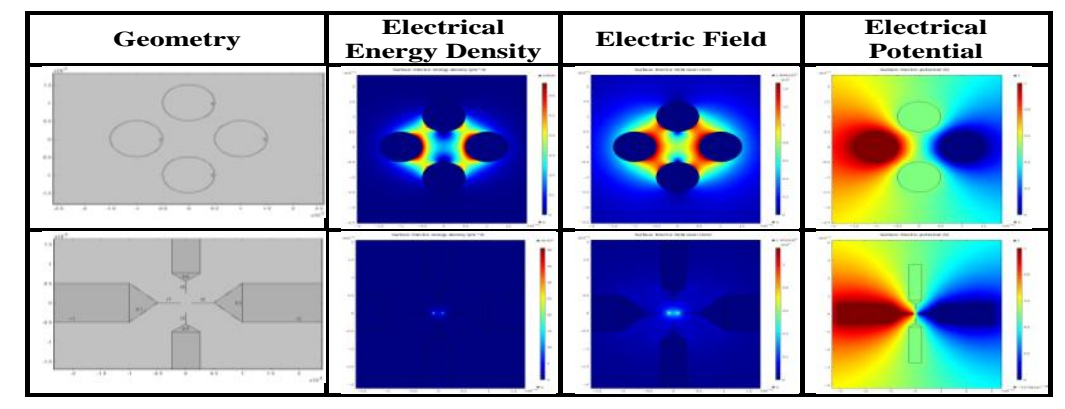

Figure 2.18: Geometry, electrical energy density, E-Field and potential of circular and triangle shapes with antenna structure

The simulations revealed that the structure shown above clearly confines the electric fields and electric energy density into a margined area away from the silver source. It has been established that devices with the antenna type of structure as shown above confines the E-field and the electric density effects to very small areas between the two antennas. Another advantage from these simulations is the ability to create many devices of different dimensions using a specific ratio of $1 / 1.5$ which corresponds to Al electrode spacing to Ag source spacing respectively. Using the aforementioned ratio and with the aid of Visio software, masks for these devices were created for the following process flow.

The process flow for device fabrication starts with a photolithography, on top of an insulator, that creates openings for Al deposition followed by a secondary photolithography step to allow for the deposition of the Ag pads. This step is followed by a third lithography which covers the large pads of the $\mathrm{Al}$ and $\mathrm{Ag}$ pads with photoresist while allowing for the deposition of a $100 \mathrm{~nm}$ chalcogenide glass film to deposit over the entire structure with the exception of the areas covered by the photoresist. This process ensures that the chalcogenide glass film is not exposed to the basic solutions that are customary in the contemporary photolithography processes since these glasses dissolve in a basic solution. Following the $\mathrm{Al}$ deposition process, a thin layer of $\mathrm{Ag}$ was deposited capping the $\mathrm{Al}$ pad, without breaking vacuum to prevent the oxidation of Al. In this manner, it is possible to fabricate this sensor using standard CMOS semiconductor fabrication processes, which would allow embedding of these devices in an IC during the Back End of Line process. The process flow sequence is presented in Figure 2.19 and the masks used for fabrication of different devices are shown in Figures 2.20 - 2.22. 


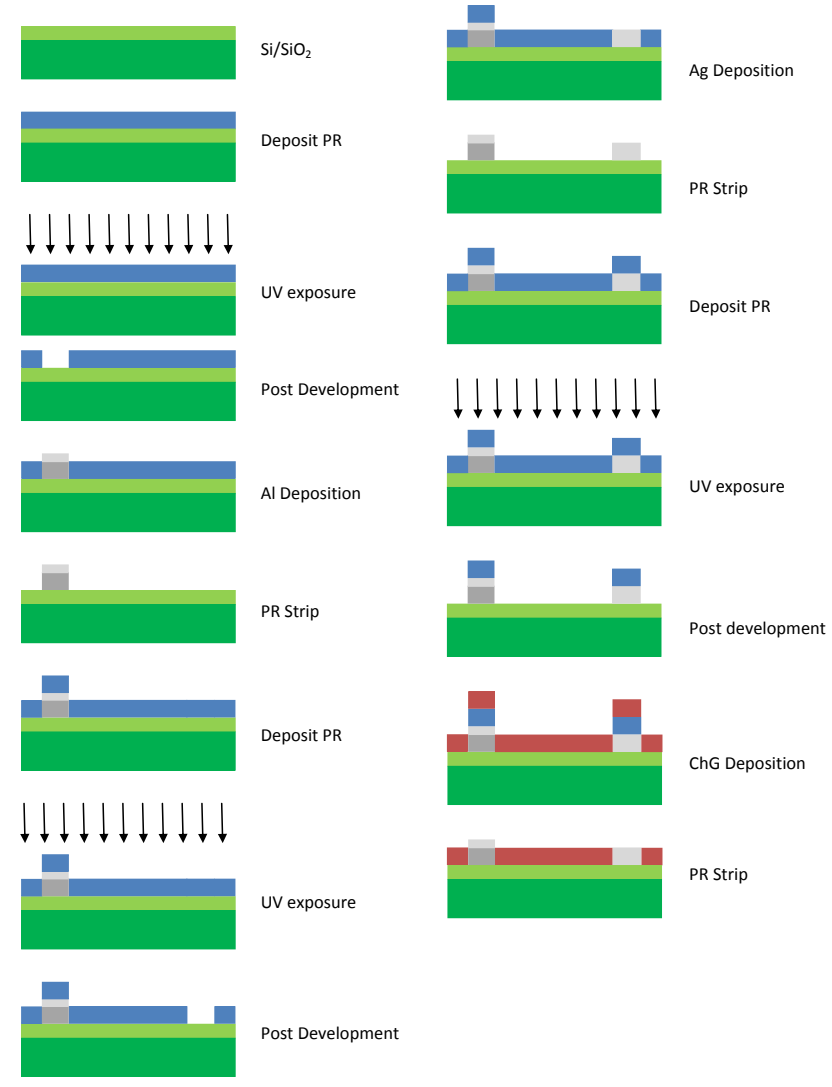

Figure 2.19: Process Flow for fabricating simulated device structure

The COMSOL simulations have revealed a specific type of electrodes for the aluminum electrodes, but the silver electrodes can be created with different geometries since the electric fields are confined to areas between the aluminum electrodes. For this reason, four types of electrode geometries (antenna, no antenna, circle and square) were created on the various masks. These masks and devices dimensions are described below.

Table 1: Four types of silver electrode geometries

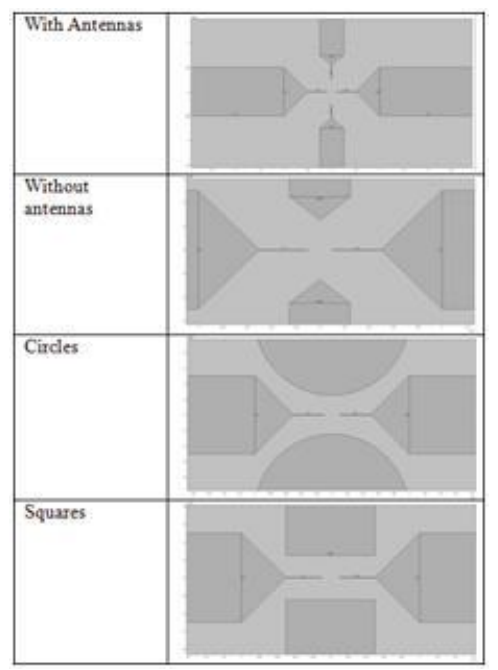


Various Al spacing ranging from $10 \mathrm{~mm}$ to $250 \mu \mathrm{m}$ were created such that are 20 devices for each type of Ag geometry. The various device dimensions are shown in the table below.

Table 2: Aluminum and Silver device spacing on the small device mask

\begin{tabular}{|c|c|c|c|c|c|c|c|c|c|c|c|c|c|c|c|c|}
\hline $\begin{array}{l}\text { Al } \\
\text { Spacing }\end{array}$ & $1 \mathrm{~mm}$ & $1 \mathrm{~mm}$ & $1 \mathrm{~mm}$ & $1 \mathrm{~mm}$ & $\begin{array}{l}.5 \\
\mathrm{~mm}\end{array}$ & $\begin{array}{l}5 \\
\mathrm{~mm}\end{array}$ & $\begin{array}{l}5 \\
\mathrm{~mm}\end{array}$ & $\begin{array}{l}5 \\
\mathrm{~mm}\end{array}$ & $\begin{array}{l}35 \\
\mathrm{~mm}\end{array}$ & $\begin{array}{l}.35 \\
\mathrm{mmm}\end{array}$ & $\begin{array}{l}.35 \\
\mathrm{~mm}\end{array}$ & $\begin{array}{l}.35 \\
\mathrm{~mm}\end{array}$ & $\begin{array}{l}.25 \\
\mathrm{~mm}\end{array}$ & $\begin{array}{l}.25 \\
\mathrm{~mm}\end{array}$ & $\begin{array}{l}.25 \\
\mathrm{rmm}\end{array}$ & $\begin{array}{l}.25 \\
\mathrm{~mm}\end{array}$ \\
\hline $\begin{array}{l}\mathrm{Ag} \\
\text { Spacing }\end{array}$ & $\begin{array}{l}1.25 \\
\mathrm{mmm}\end{array}$ & $\begin{array}{l}0.625 \\
\mathrm{~mm}\end{array}$ & $\begin{array}{l}0.300 \\
\mathrm{~mm}\end{array}$ & $\begin{array}{l}0.15 \\
\text { mom }\end{array}$ & $\begin{array}{l}125 \\
\mathrm{~mm}\end{array}$ & $\begin{array}{l}625 \\
\mathrm{~mm}\end{array}$ & $\begin{array}{l}300 \\
\mathrm{~mm}\end{array}$ & $\begin{array}{l}.15 \\
\mathrm{~mm}\end{array}$ & $\begin{array}{l}1.25 \\
\mathrm{mmm}\end{array}$ & $\begin{array}{l}625 \\
\mathrm{mmm}\end{array}$ & $\begin{array}{l}300 \\
\mathrm{~mm}\end{array}$ & $\begin{array}{l}15 \\
\mathrm{~mm}\end{array}$ & $\begin{array}{l}1.25 \\
\mathrm{~mm}\end{array}$ & $\begin{array}{l}625 \\
\mathrm{~mm}\end{array}$ & $\begin{array}{l}.300 \\
\mathrm{~mm}\end{array}$ & $\begin{array}{l}.15 \\
\mathrm{~mm}\end{array}$ \\
\hline
\end{tabular}

Table 3: Aluminum and Silver device spacing on the big device mask

\begin{tabular}{|l|l|l|l|l|}
\hline A1 Spacing & $10 \mathrm{~mm}$ & $10 \mathrm{~mm}$ & $5 \mathrm{~mm}$ & $5 \mathrm{~mm}$ \\
\hline Ag Spacing & $12.5 \mathrm{~mm}$ & $6.25 \mathrm{~mm}$ & $6.25 \mathrm{~mm}$ & $1.25 \mathrm{~mm}$ \\
\hline
\end{tabular}

Therefore, using two wafers, and the completion of one process flow, 80 different devices can be fabricated. The devices were separated into two parts, large devices and small devices, such that all 80 devices can fit onto the fewest number of 4" wafers. Various masks were created that enable the creation of these devices, of which the masks corresponding to the creation of small devices are shown below.

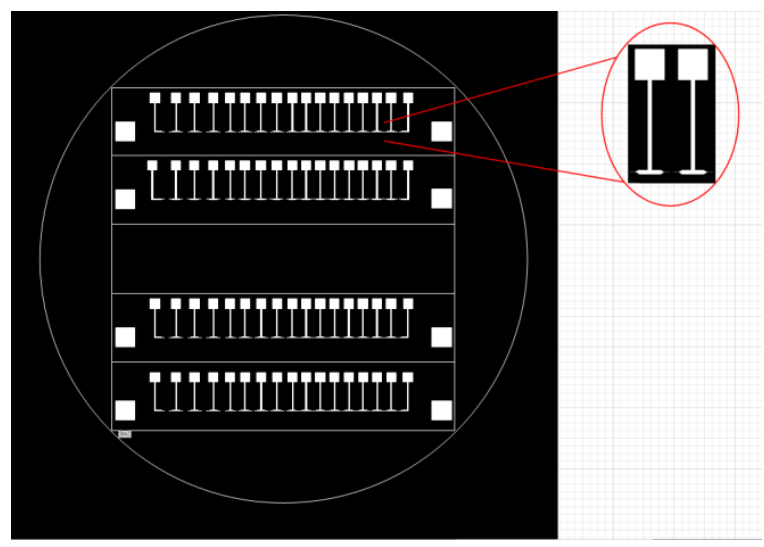

Figure 2.20: Mask for creating Al electrodes for small devices

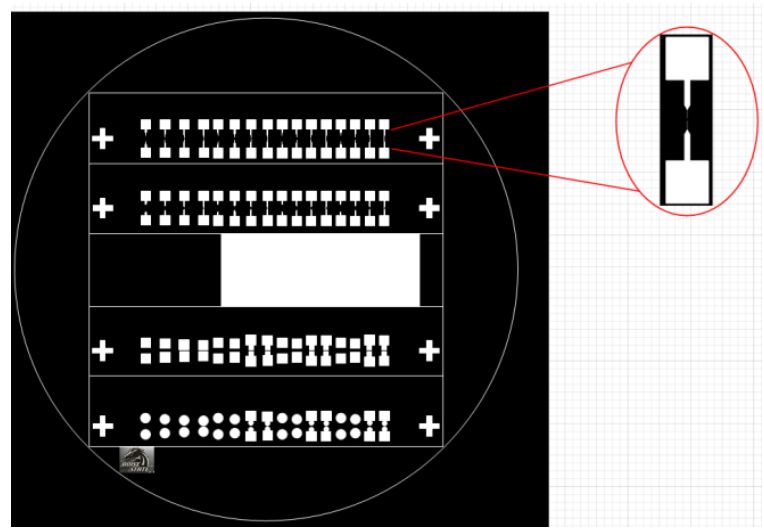

Figure 2.21: Mask for creating Ag electrodes for small devices 


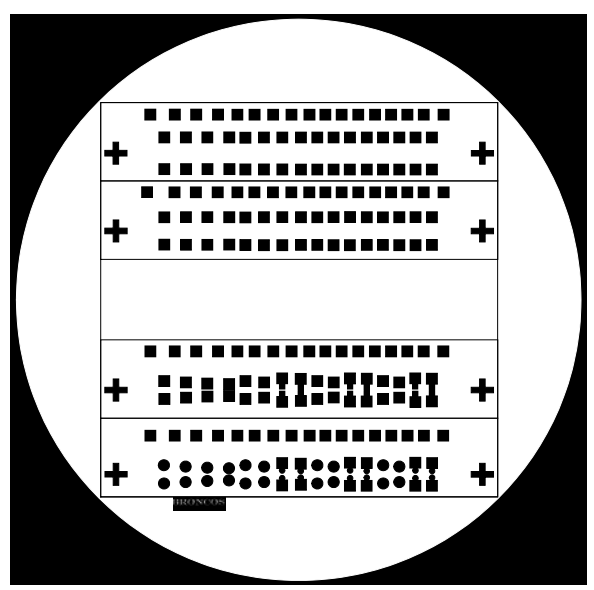

Figure 2.22: Mask for depositing chalcogenide films for small devices

Data about the radiation-induced performance of these devices are presented on Figure 2.21. We established that for these devices best test data are collected at $2 \mathrm{~V}$ since at this voltage the devices performance stabilizes. Additionally, due to the investigation into isolating the electric fields, this increased voltage does not play a role in the silver diffusion so it is not harmful to the device behavior.
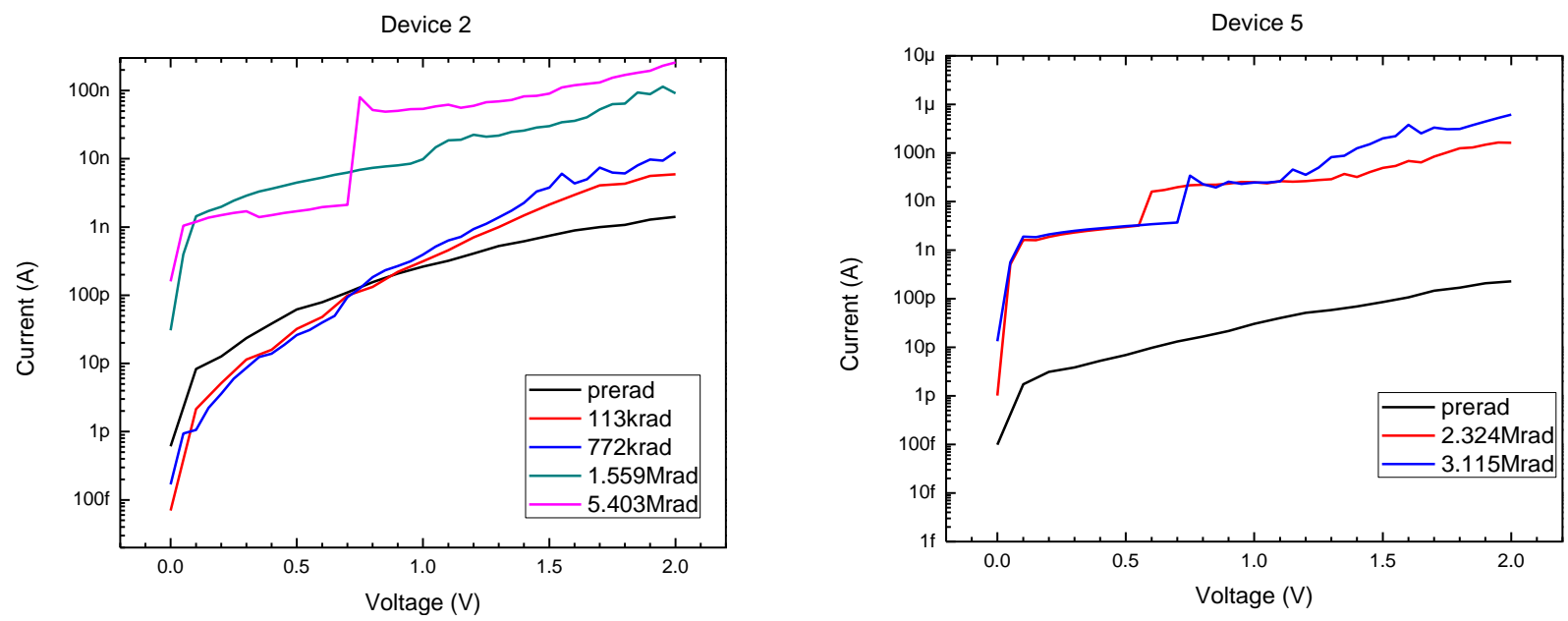

Figure 2.23. IV curves of devices irradiated with different doses a) $\mathrm{Ge}_{20} \mathrm{Se}_{80}$; b) $\mathrm{Ge}_{40} \mathrm{Se}_{60}$ glasses

As is seen measurable difference in the devices performance at different radiation doses can be created. The $\mathrm{Ge}_{40} \mathrm{Se}_{60}$ glass can be used as shown also in the previous data for sensing higher radiation doses.

\subsection{Vertical Devices with CMOS Compatible Size}

Vertical devices were created with the presumption that a metal bottom electrode underneath the $\mathrm{Ag} / \mathrm{Ge}-\mathrm{Se}$ device can enhance the sensor performance characteristics. These structures were fabricated with the following materials - Nickel (Ni) as the cathode, GeSe as the solid electrolyte and Silver (Ag) as the Anode. A cross section and a top view of these dveices are shown on figure 2.24 . 


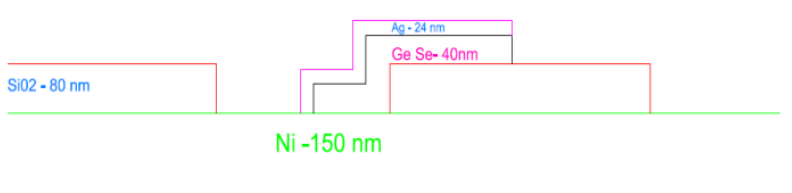

Si-Substrate

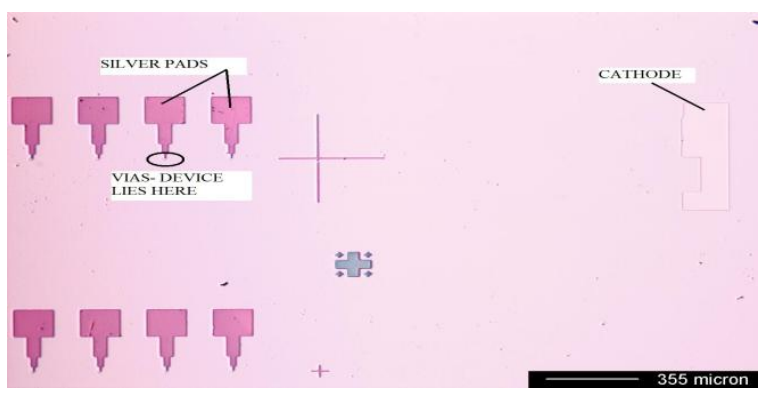

Figure 2.24. Vertical devices with CMOS compatible dimensions a) Cross section b) top view

Data about the performance of these devices as a function of radiation are presented on figure 2.23. In fact, the vertical resistance (between the Ag top and $\mathrm{Ni}$ bottom electrodes) increases with increasing dose. This is due to an increase in the resistivity of the doped region between the top and bottom electrodes by a reduction in $\mathrm{Ag}$ concentration even as the area of the doped region between the top and bottom electrodes is increased by the lateral diffusion of the Ag. These devices did not show any significant change in resistance during annealing intervals. Although resistance of these devices increased with increasing dose, it is indicated that the opposite effect can be achieved (i.e. decrease of resistance with TID) by fabricating devices with thicker Ag electrodes $(\sim 150-300 \mathrm{~nm})$. These results show that using metal substrates could improve sensor range and resolution.

Previous studies on photodoping of $\mathrm{ChG}$ have shown that depositing $\mathrm{ChG}$ on a metallic substrate increases the rate of Ag lateral diffusion $[15,16]$. Since the electronic conductivity is low in ChG materials, the electrons can pass laterally through metallic substrate and therefore enhancing the reaction rate at $\mathrm{Ag}$ and $\mathrm{ChG}$ interface. As a result, sensor response time can also be improved by having metal cathode with higher electronegativity's as compared with Ag.
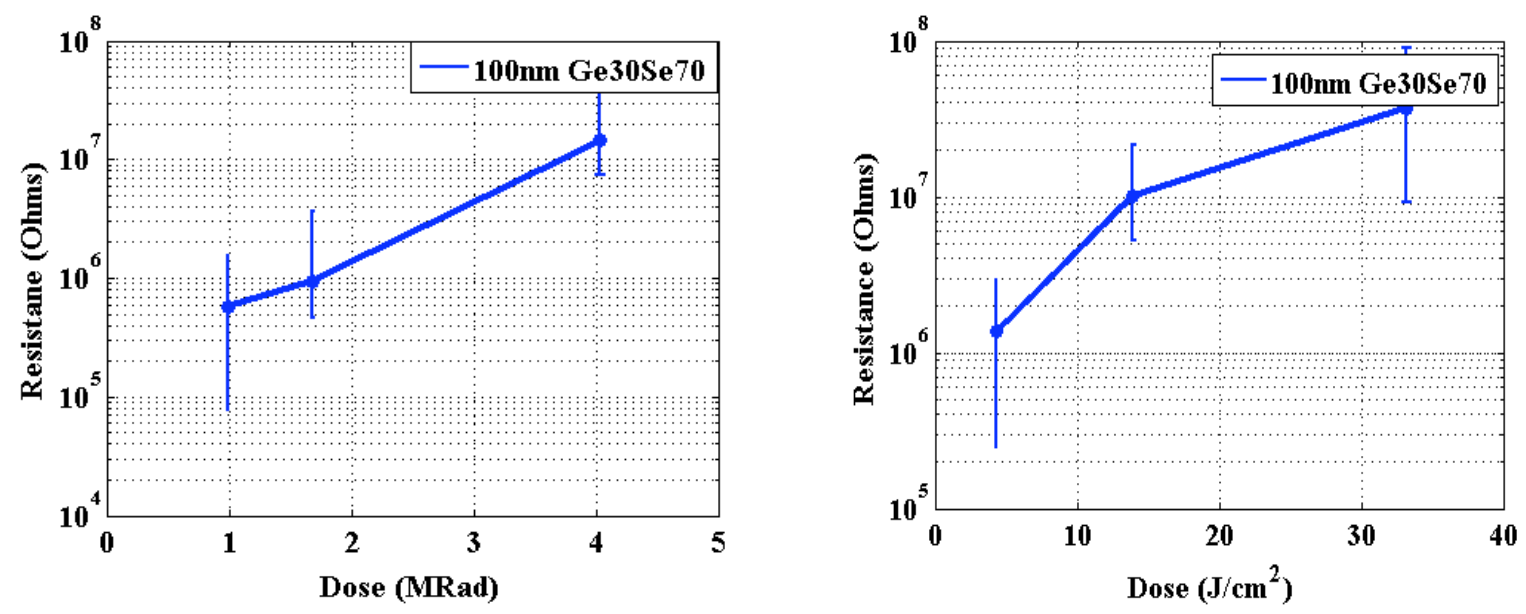

Figure 2.25. Vertical devices: Resistance as a function of (a) gamma TID and (b) total UV dose exposure of $\mathrm{Ag} / \mathrm{Ge}_{30} \mathrm{Se}_{70} / \mathrm{Ni}$.

From UV and gamma irradiation experiments, the sensor performance factors $\left(\mathrm{D}_{\mathrm{th}}, \mathrm{R}_{\mathrm{th}}\right.$, $\mathrm{D}_{\text {sat }}, \mathrm{R}_{\text {sat }}$ ) depends on: ChG thickness, ChG composition, Ag thickness, distance between the two electrodes, substrate underneath the device (insulator, metal conductors, semiconductors), electrode configuration (bus bars, interdigitated fingers, array or ring electrodes), radiation sources (intensity, dose rate), etc. 
The thickness of $\mathrm{ChG}, 20 \mathrm{~nm}$ or less, has shown to be distinguishing parameter for the $\mathrm{Ag}$ lateral diffusion. Thin $5 \mathrm{~nm} \mathrm{ChG}$ film has shown to have faster saturation rate of $\mathrm{Ag}$ diffusion than $20 \mathrm{~nm} \mathrm{ChG}$ film. The ChG composition has shown to have an effect on Ag diffusion rate, increase in the Se content, leads to increase in diffusion rate and higher Ag incorporation. Ag photodissolution was also observed in other ChG materials such as Ge-Te, Ge-S, As-S, As-Se, As-Te, As-S-Te and As-Se-Te [15-18], and the maximum diffusion occurs along the composition of lowest density. Studies have shown that the diffusion in sulfide glasses is slower than selenide glasses $[16,19]$. As a result of changing the thickness and composition of ChG materials, the dynamic range of $\mathrm{D}_{\text {th }}$ and $\mathrm{D}_{\text {sat }}$ can be varied for different total ion doses.

Silver thickness determines the amount of $\mathrm{Ag}$ dissolved in $\mathrm{ChG}$ and the lateral $\mathrm{Ag}$ diffusion distance, which influences the $\mathrm{R}_{\text {th }}$ and $\mathrm{R}_{\text {sat }}$ of the sensor. Silver can be replaced with widely used interconnect metal in the semiconductor industry, $\mathrm{Cu}$, as $\mathrm{Cu}$ has shown similar photodiffusion results in ChG $[15,20]$. Distance between the electrodes shows linear dependence on $\mathrm{D}_{\text {th }}$ of the sensor; hence, it is supposed that lower $\mathrm{D}_{\text {th }}$ can be achieved by reducing the electrode distance to ' $\mu \mathrm{m}$ ' or 'nm' range.

\subsection{CMOS compatible device design with Ag film on top for measurement of increased resistance as a function of radiation}

The primary design approach that has been pursued for most of the program, i.e., our baseline technique, has been determined to be most adequate for measuring very high doses of ionizing radiation. The mechanism for the baseline technique is decreased impedance across a chalcogenide glass $(\mathrm{ChG})$ film as ionizing radiation exposure facilitates the diffusion of metal ions into the $\mathrm{ChG}$ matrix. While the results obtained for this high dose radiation sensor have been promising, the team decided to simultaneously develop an alternative sensing approach capable of detecting much lower levels of ionizing radiation dose. This alternative design measures increased impedance across two terminals with an increase in the radiation dose. When energy is deposited by ionizing radiation (e.g. ${ }^{60} \mathrm{Co}$ gamma rays), ions in a continuous active metallic layer, patterned between the terminals, diffuse into the $\mathrm{ChG}$ matrix, which breaks the bridge between the two electrodes thereby increasing the resistance across the structure. Preliminary investigations of the new device's response characteristics show much high levels of radiation sensitivity at low levels of total ionizing dose $(<50 \mathrm{krd}(\mathrm{Ge}))$.

The test structures was designed with the following principle in mind: creation of a low resistance (continuous) silver film prior to irradiation on the surface of the devices and creation of a high resistance (discontinuous) silver film post irradiation on the device surface, so that the surface resistance changes as a function of the dissolution of the Ag film. The idea of this structure is shown in Figure 2. 26. 


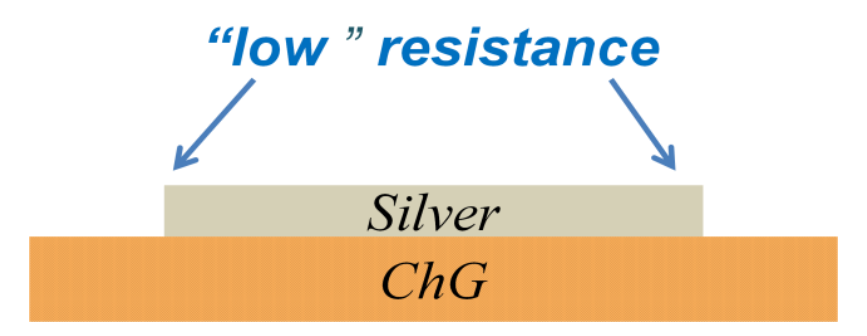

\section{radiation}

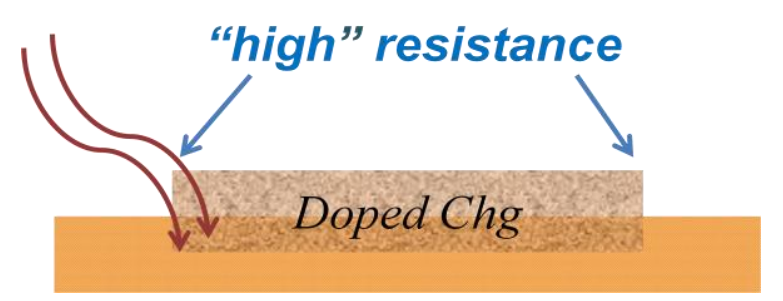

Figure 2.26. Principle of ChG based sensor operation

These structures are designed such that, after processing we are measuring the resistance of a patterned silver pad on blanket $\mathrm{Ge}_{30} \mathrm{Se}_{70}$ which corresponds to a low resistance state compared to a high resistance state post irradiation relating to the resistance of the chalcogenide film doped with silver.

\section{Mask design of test structures}

A mask was designed using AUTOCAD software to produce the following test structures. The mask was designed using positive photoresists therefore; dark field masks were created in this aspect. Nickel was used as the electrode material on top of the silver pad. The inert electrodes were created using nickel because it is inert in nature and can be easily evaporated at moderate temperatures. Silver cannot be probed directly as silver on top of $\mathrm{Ge}_{30} \mathrm{~S}_{70}$ because Ag can diffuse at room temperature or when exposed to radiation, which will not create stable electrodes. There are two masks required for the processing of these test structures.

\section{Mask 1- Silver pad mask}

Initially, a blanket layer of $\mathrm{Ge}_{30} \mathrm{Se}_{70}$ film was deposited on oxidized silicon wafers. This step was followed by depositing silver in specific areas of the film with the aid of Mask 1 . This mask was designed to create rectangular pads and when the following lithography step has been performed, the subsequent Nickel electrodes are placed on top of the silver pads. In this manner, two adjacent Ni electrodes are connected together with a rectangular patterned silver film. The layout of these pads is as shown in Figure 2.27. The corners of the pads have been rounded off to avoid high electric field at that the corners. The radius is $2 \mu \mathrm{m}$ and this is consistent throughout the mask design for all features with corners. 

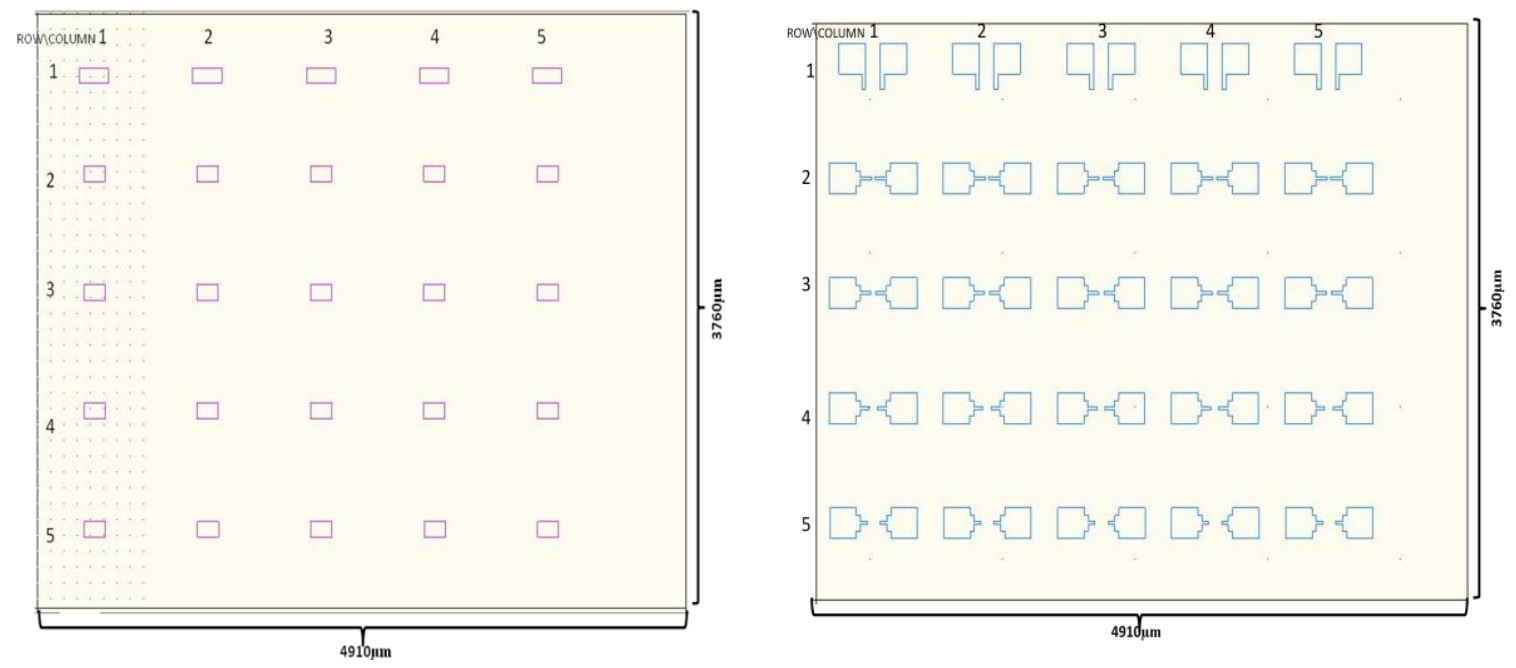

Figure 2.27. Layout of masks a) silver pad mask; b) nickel electrode mask

The pink rectangular pad is the pattern for creating the continuous silver layer placed on top of the $\mathrm{Ge}_{30} \mathrm{Se}_{70}$ blanket film. These have been spaced apart such that we have enough room for depositing electrode material (Ni) on top. The width of each rectangle is $100 \mu \mathrm{m}$, the length of the pads in row 1 are $220 \mu \mathrm{m}$ and the pads in rows $2-5$ is $160 \mu \mathrm{m}$. Device dimensions, and spacing have been illustrated in figure 2.26.

There are two different structures on the mask- type A (with the electrode fingers parallel to one another) and type B (with the electrode fingers along the same line facing one another). There are five type A structures along the first row, the spacing between the fingers is fixed at $100 \mu \mathrm{m}$. The rows 2-5 consist of devices with type B structures. The spacing between the fingers increases row wise $(30,40,60,100 \mu \mathrm{m})$.

Type A and type B structures
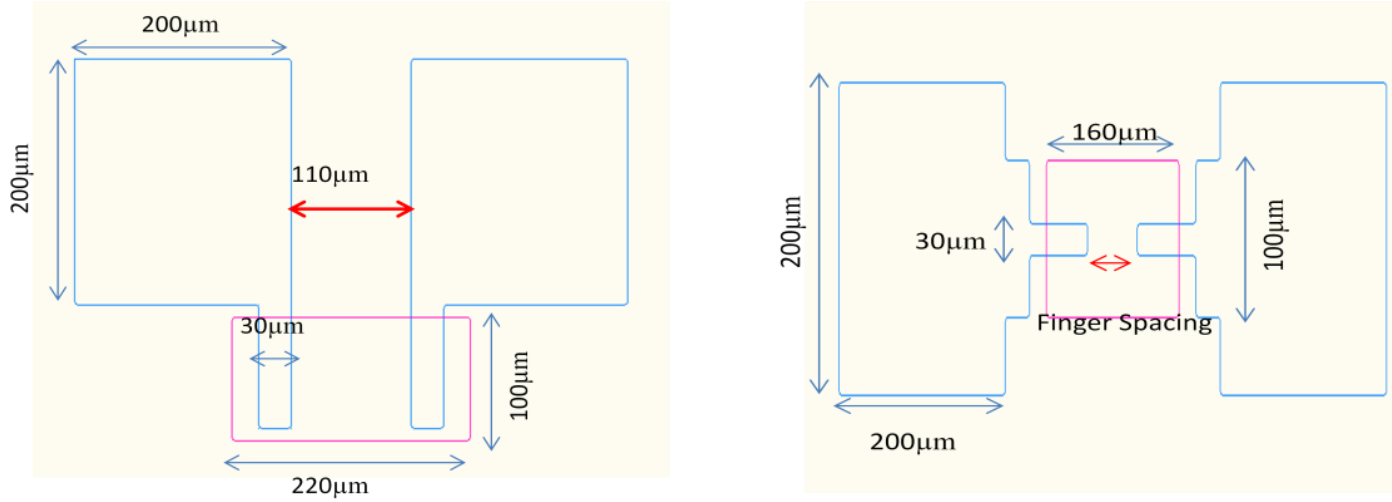

Figure 2.28. a)Dimensions of type A structure; b) Dimensions of type B structure

The cross-sections of type A and type B structure have been shown in the Figure 2.29.a) and b) respectively. 


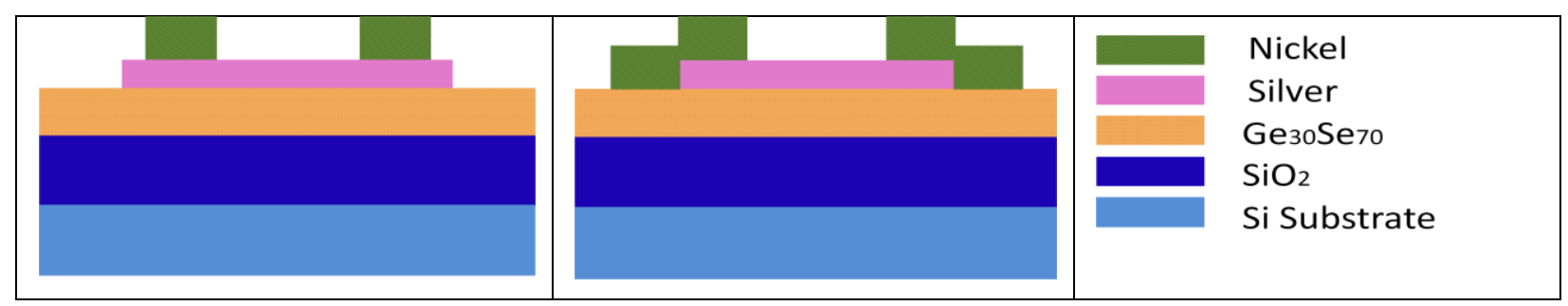

Figure 2.29. Device cross sections (cut-line made in the middle of the device): a) type a and b) type b) structure.

Optical images of the structures were taken using the Axiophot (high resolution optical microscope). Figures $2.30 \mathrm{a}$ ) and b) illustrate the images of type A and type B structures. Images are taken soon after the structures are manufactured and they help in early analysis of the test structures. It was observed the appearance a halo around the devices corresponding to silver diffusion, which indicates that some silver diffusion into the $\mathrm{ChG}$ was taking place at room temperature possibly due to the processing steps involved.
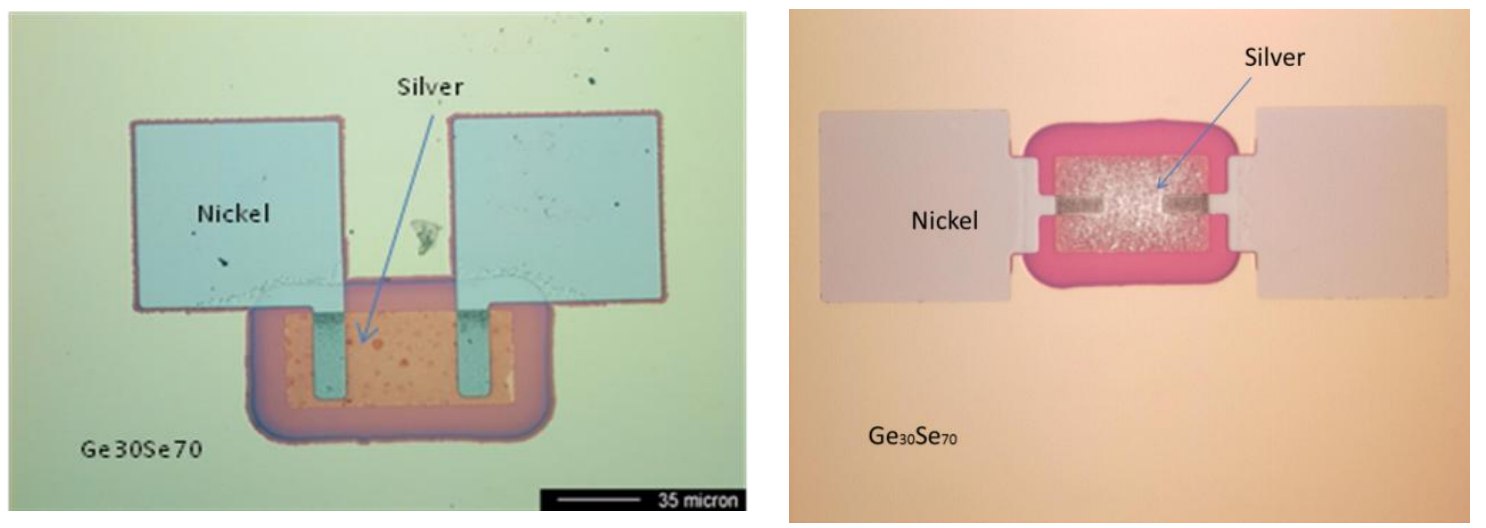

Figure 2.30. Optical images of the devices: a) type a and b) type B

Electrical characterization of the test structures:

The electrical performance of the test structures was accomplished through measuring of the current-voltage characteristics of the devices using a computer controlled Agilent 4156C semiconductor parameter analyzer. The substrate of the wafer was left floating during the measurement. These measurements were conducted on a probe station with guarded co-axial cables. The experiment was carried out with minimal ambient light and there was no microscope light on the structures during the measurement. All these precautions were necessary in order to avoid introduction of an additional influence to the devices performance created by light.

The evolution of current as a function of dose and time is shown in Figure 2.31. for type A structures with $110 \mu \mathrm{m}$ length between electrodes. In black are represented the control devices, in red are represented devices of type A from sample II on which current are measured for low total irradiated dose steps, in green are represented devices of type A from sample III that were tested at higher radiation dose than the devices on sample II. The current presented here are for 5 devices of type A for each of the three dies tested. 


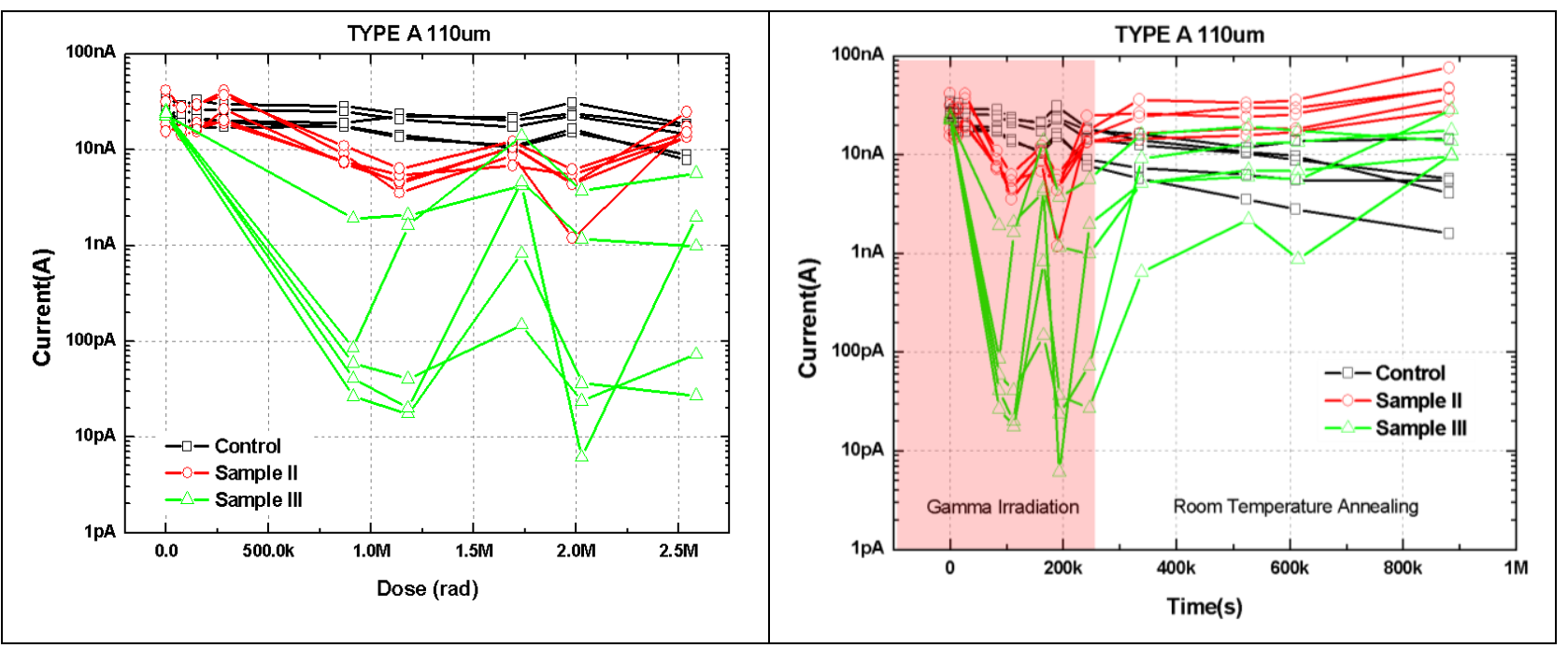

Figure 2.31. Evolution of current as a function of: a) dose for type A structures; b) time for type A structures

The current evolution as a function of time is shown in Figure $2.31 \mathrm{~b}$ ) for similar type A structures. In black is the data corresponding to the control devices, in red are devices of type A from sample II measured at low total irradiated dose steps at the beginning; in green are devices of type A from sample III measured at higher radiation doses. The first $250 \mathrm{kseconds}$ are the evolution of the current due to the exposure to gamma rays (highlighted with a pink area), while the room thermal annealing period goes from 250 kseconds to 1 Mseconds. This annealing period is referred to placing the devices in a dark environment at room temperature and measuring the device performance after discrete time intervals after the radiation experiment.

The current level lowers with radiation dose up to a gamma dose of $1 \mathrm{Mrad}$. The magnitude of the current variation is different for devices on dies 2 and 3. This can be due to an impact of the several measurements conducted on sample II at lower total doses, while sample III remained in the irradiator until $912 \mathrm{krad}$. Current on the control devices decreases by 1 order of magnitude (black curve) during the duration of the experiment. For the low total dose devices (on sample II), the current was constant after several small dose steps, but the current begins to decrease at the same dose as the devices on sample III which remained in the gamma cell. The current level for sample III (taken out after 912krad) is at a much lower level than that obtained on devices on die II. This suggests that devices have to be exposed to a certain value of dose (at once) to see the drop in current, or that performing repeated electrical characterization has an impact on the response of the devices. Current of devices irradiated decreases after exposure, but it can be seen on Figure 2.31 that the current recovers to a level equivalent and higher to its initial level after 7 days of room temperature annealing.

The evolution of current as a function of dose for the B type of devices is shown in Figure 2.32 for type structure with $110 \mu \mathrm{m}$ spacing between the fingers: In black are represented the control devices, in red are represented devices from sample II, in green are represented devices of type B from sample III in green which were kept in the chamber for longer intervals. 

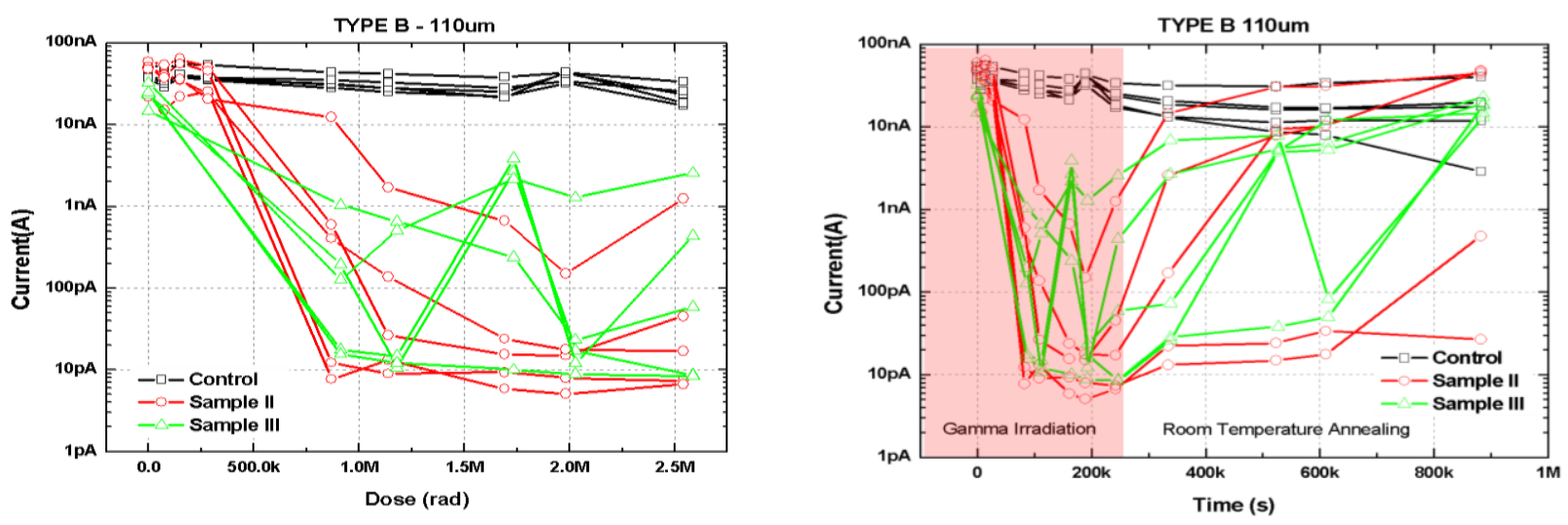

Figure 2.32. Evolution of current as a function of: a) dose for type B devices; b) time for type B devices

It is observed on Figures 2. $32 \mathrm{a}$ ) and b) that current decreases slightly on the control structures (black curve) which might be the result of repeated measurements. Current measurements on the structures from sample II do not decrease with dose for the lower dose steps. At higher total doses, the current measured on these structures decreases by 2- and in some cases 3 orders of magnitude. Current measurements on the devices from sample III decreases with dose but increase back to the initial value when sample is left at room temperature for several days. Some devices exhibit a current measurement after the room temperature annealing which is as high as their pre-irradiation current level. This self-recovery effect can be used for reuse of the devices once the exact annealing time necessary for a full recovery to the initial condition of the studied structures is determined.

In summary: the following observations were made during the course of the experimenta low pre-irradiation resistance level, increase in resistance with radiation dose and recovery of resistance to pre-irradiation level post exposure at room temperature after specific period. We believe that these effects are closely related to the devices structure and the chalcogenide glass composition used for formation of the studied devices.

The resistance value close to $1 \mathrm{M} \Omega$ obtained prior to irradiation as opposed to resistance in the order of few tens of ohms is the initial characteristic of these devices. A metallic layer of silver corresponds to a sheet of very low resistivity with resistance in the order of few tens of ohms. This high value of resistance can be due to the processing steps that the structures undergo. Steps such as thermal evaporation of films can cause thermal diffusion of silver into the ChG. Agglomeration of thin silver films can also take place. Agglomeration results in reduction of inter-facial energy. This results in loss in continuity of the silver film above the ChG. Silver has high mobility in the $\mathrm{ChG}$, this can cause some diffusion to take place at room temperature. All the three processes mentioned above result in the loss of a continuity of the silver film above the $\mathrm{ChG}$ resulting in a higher pre-irradiation resistance level.

It was observed that there was change in resistance (increase) with radiation dose. There was a three orders of magnitude change in the resistance level with radiation. This change was a function of the total irradiated dose. It was noticed that at smaller dose steps, there was no change in resistance of the test structure. The change was a function of the total irradiated dose. When radiation stress is applied, there is generation of electrons and holes in the ChG film. The generation of electrons and holes in the $\mathrm{SiO}_{2}$ is not covered within the scope of this work. The $\mathrm{Ag}^{+}$ions combine with the electron and are neutralized creating atomic silver. Additionally, silver can bond with selenium atoms creating $\mathrm{Ag}_{2} \mathrm{Se}$ as shown in the chemical formula below. 


$$
\begin{gathered}
\mathrm{Ag}^{+}+\mathrm{e}^{-}->\mathrm{Ag} \\
2 \mathrm{Ag}^{+}+\mathrm{Se}^{-2}->\mathrm{Ag}_{2} \mathrm{Se}
\end{gathered}
$$

$\mathrm{Ag}_{2} \mathrm{Se}$ clusters increases the density of the film [21, 22] and as a result we have less continuous film of silver on top, this causes the drop in resistance when exposed to radiation.

Resistance recovers to a value close to the pre-irradiation resistance level when the irradiated test structures are kept at room temperature was may be the most interesting effect occurring in the measured structures. This could be due to photo stimulated deposition [23] of silver on the surface at room temperature. The resistance measured is that of the silver film on top of the ChG. Silver ions migrate towards the surface resulting in a lowering of resistance. The force behind silver migration could be the photo excited electrons at the interface which attracts the excess silver ions in the glass by the electric field. There can also be photo-induced holes diffusing into the interior of the ChG glass and excess silver ions counter flow to the hole motion [24]. This results in silver being deposited at the surface of the film resulting in a lowering of resistance close to the pre-irradiation resistance level.

Recovery was also noticed in the case of some structures that stayed in the irradiator after being tested. The measurement time is long enough ( 1 hour in total for all die) for the recovery process to start. The structures do not stay at the low level of current and start recovering once they are placed back in the irradiator. In-situ testing can prove helpful in such situations.

It is of interest to understand how the thickness of the chalcogenide film affects the devices performance. The output (resistance) was plotted for structures from for radiation dose of $800 \mathrm{krad}$ as shown in Figure 2.33. It was observed that for both the type of thickness characteristic fo the samples from batch $1(40 \mathrm{~nm})$ and $2(80 \mathrm{~nm})$, change in dose/ $\mathrm{dB}$ is close to $400 \mathrm{krad}$. This suggests that sensitivity is independent of the thickness of the silver film.

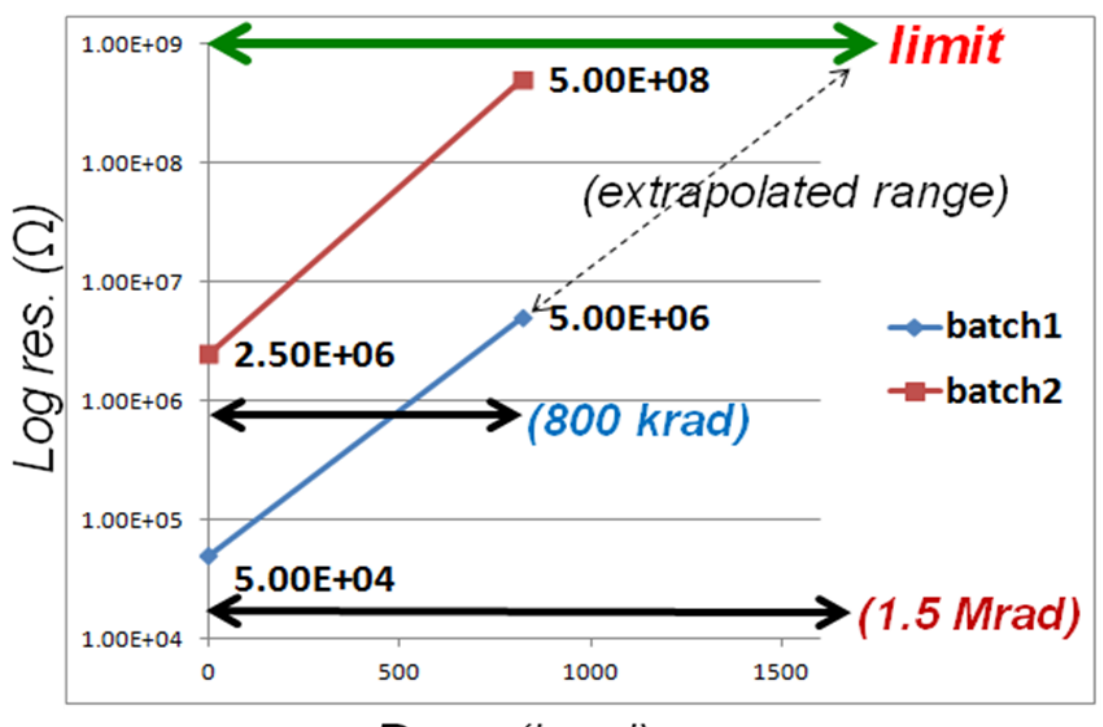

Dose (krad)

Figure 2.33. Resistance plotted as a function of dose for batch 1 and 2

It can also be seen from Figure 2.33 that in the case of batch 1 with lower pre-irradiation resistance level, there is an extended range of operation for the sensor. The range is limited in the case of batch 2 at $800 \mathrm{krad}$. The limit is set by the maximum resistance that can be measured with the instrument. 


\section{Summary}

As a result of the work on the project, the entire program as presented in the proposal has been fulfilled. The outcome of the research sheds light on the basic effects related to creation of radiation induced effects and $\mathrm{Ag}$ diffusion in structures containing chalcogenide glasses in a proximity to a Ag source. We created 6 different structures and studied the performance of each type of radiation sensor design, which have the advantage of being low cost, high performance microelectronic devices that react to $\gamma$ radiation to produce an easily measured change in electrical resistance. These radiation sensors are two-terminal micro devices with an active region consisting of a chalcogenide glass $(\mathrm{ChG})$. Exposure to ionizing radiation stimulates radiation induced effects (RIE) in the active region which promotes silver (Ag) diffusion and incorporation in the $\mathrm{ChG}$, thereby reducing the material's resistivity. Since these devices are based on amorphous films, they can be fabricated on flexible and non-planar substrates which will increase their range of application. This approach is characterized by completely new principles of operation that offer low power consumption, compatibility with integrated circuit fabrication, and operational reversibility which allows for calibration and reuse. Since the family of the chalcogenide glasses includes a large number of materials, there are extended possibilities to tailor the sensitivity of the sensor to particular use situations. Specifically we would like to underline the following results:

1. The most important representatives of the Ge-S and Ge-Se chalcogenide glasses have been synthesised ranging from chalcogen rich (containing 80 at. \% chalcogen) to Ge rich (containing 40 at. \% Ge) glasses.

2. Thin films and device structures have been created from various chalcogenide thin film compositions and radiated using $\gamma$ and $\mathrm{x}$ rays.

3. The radiation induced effects in the bare films and device structures have been studied with Raman spectroscopy, XRD, EDS, AFM and XPS. As a result of these studies a strong composition/structure dependence of the effects has been established showing that in the chalcogen rich films, the underlying effects are predominantly related to the formation of electron-hole pairs as a result of radiation, while in the case of Ge rich films, structural reorganization occurs which has been registered by the Raman studies.

4. The radiation effects accompanied with Ag diffusion are composition/structure dependent - the chalcogen rich films are more sensitive and attract Ag at low radiation doses, so that they are an attractive candidate for personal dosimetry; films containing around $30 \% \mathrm{Ge}$ are less sensitive since there is not a strong driving force for Ag to diffuse within them, but show quite stable performance; the Ge rich films are less sensitive at low radiation doses, but at high doses but they retain the performance well and are suitable for measuring high radiation doses. We suggest that this is related to the structural reorganization occurring within the Ge-rich films at these radiation conditions. Mainly the breaking of the weak Ge-Ge bonding available in such films, as well as the specific structure in which both Ge and the chalcogen are threefold coordinated which leads to good opportunity for Ag diffusion.

5. In terms of devices design and testing, we created 6 different types of devices with variety of performance abilities. Specifically, the following device structures have been successfully fabricated and studied:

a. Lateral devices with two electrochemically inert electrodes - depending on the composition of the chalcogenide glass and the radiation conditions they can result in up to 2 orders of magnitude reduction in the resistance as a result of radiation. 
They have been wire-bonded and packaged for testing. Examples of these devices are presented below:
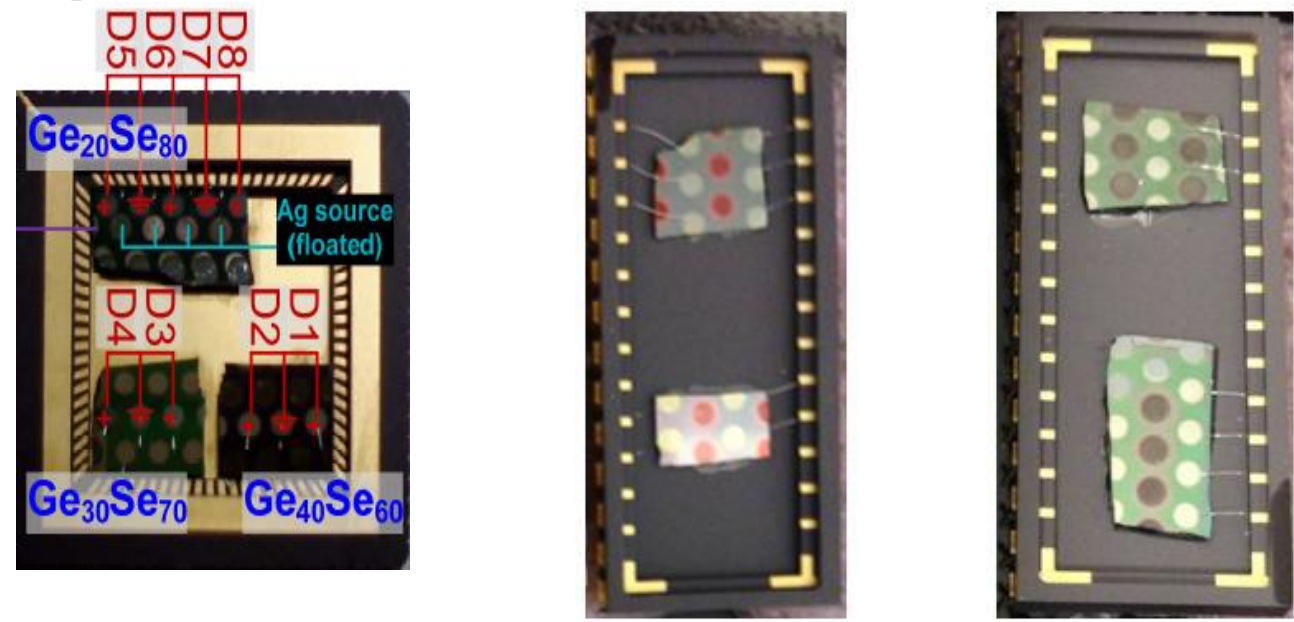

b) Lateral devices with two Ag electrodes - the ChG film sandwiched between $\mathrm{Ag}$ array electrodes and an inert metal substrate can be used to collect multiple cumulative 2D distribution of absorbed dose in a single irradiation exposure, since both radiation sensitive vertical and lateral Ag diffusion has been observed on these vertical structures. The Ag-ChG array detectors can provide alternative solution for measuring individual quantitative dose quanta at each detector point and generate a 2D contour map of dose distributions. They also have been wirebonded and packaged as shown below for improved testing results.

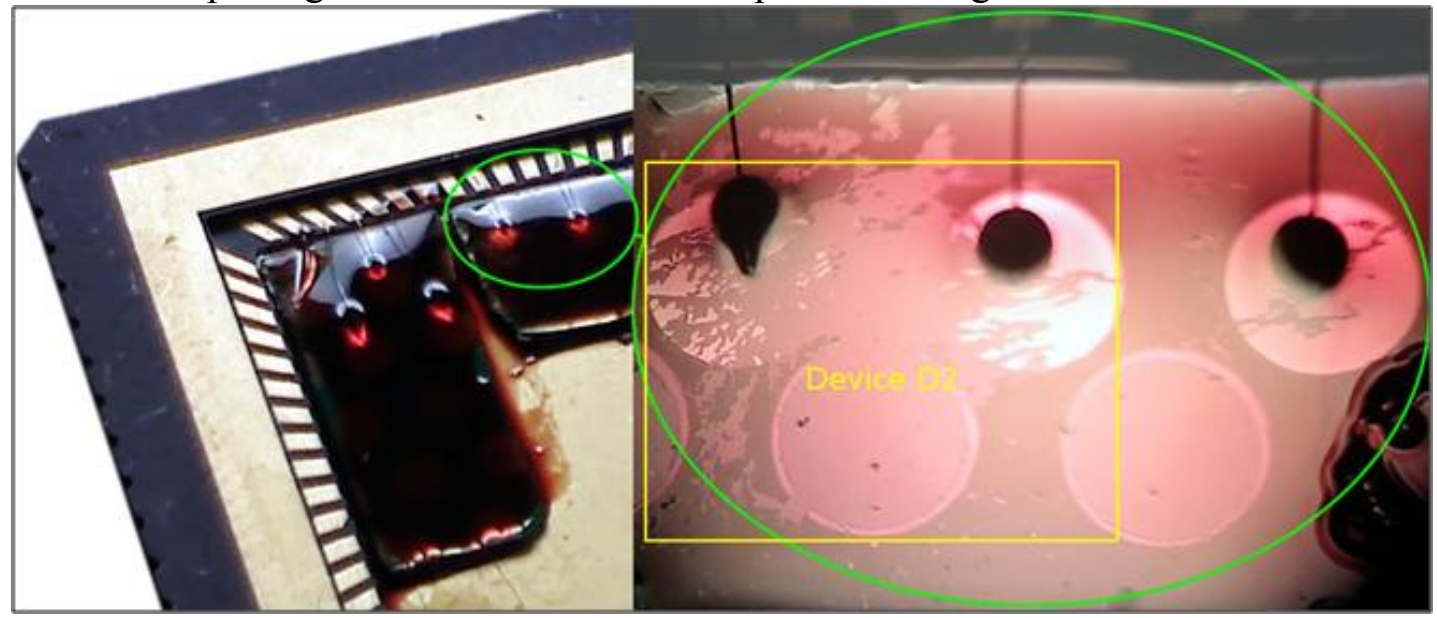

c) Layered devices - they are modeling using Silvaco Atlas and the impedance measurements helped in understanding the electrical characteristics and equivalent electrical circuit model in order to design an appropriate circuit for embedding these devices within CMOS circuit for measurement of the radiation dose using electrical signal. 

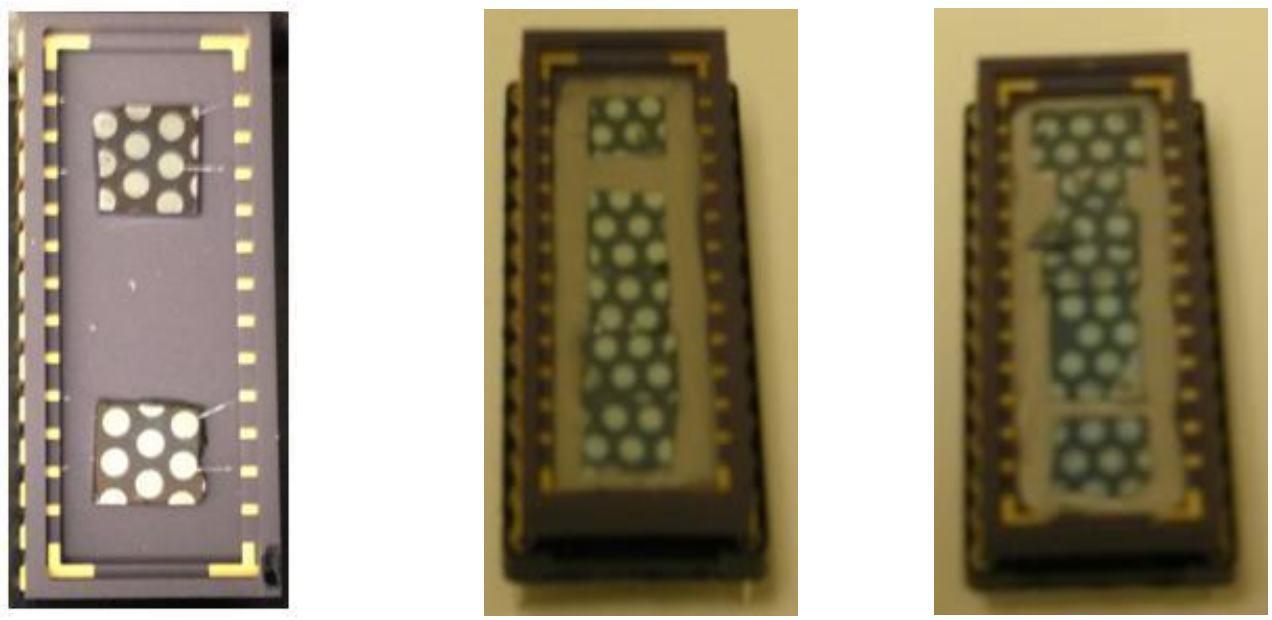

d) Small devices were modeled with Comsol - these studies defined the structures in which the influence of the electrical field applied for measuring the radiation induced $\mathrm{Ag}$ diffusion does not interfere with the measured structure for a precise radiation measurement data and real time measurements application. These devices were also wire bonded for improved measurement data as shown below for different model structures.
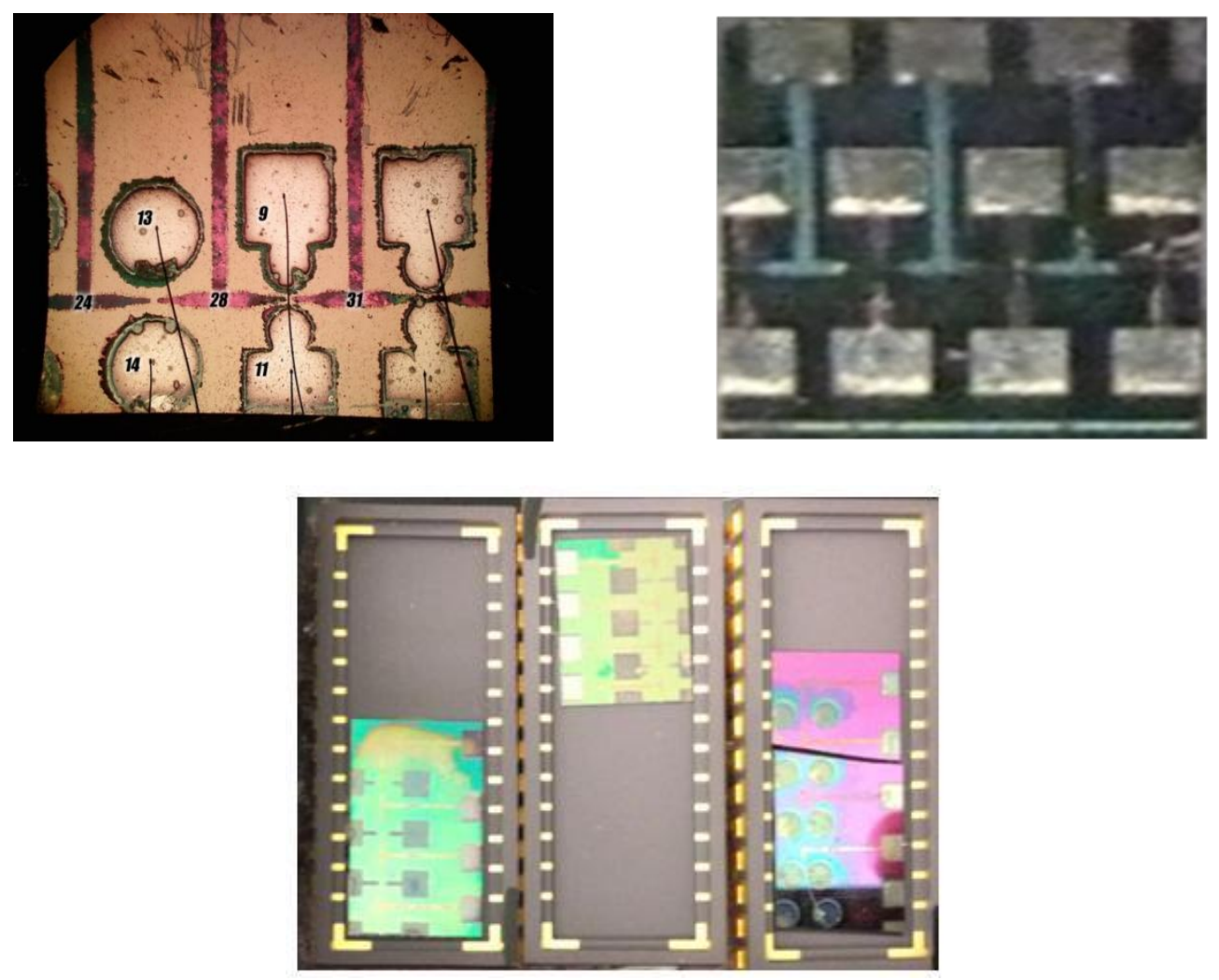

e) CMOS compatible vertical devices - their performance occurred in increased resistivity as a result of radiation, due to the device structure where the Ag 
diffusion depletes the position between the two measuring electrodes. The size of these devices allows their immediate incorporation into a CMOS integrated circuit. The small size of the devices contributes also to stable performance. Example of these devices is shown below.

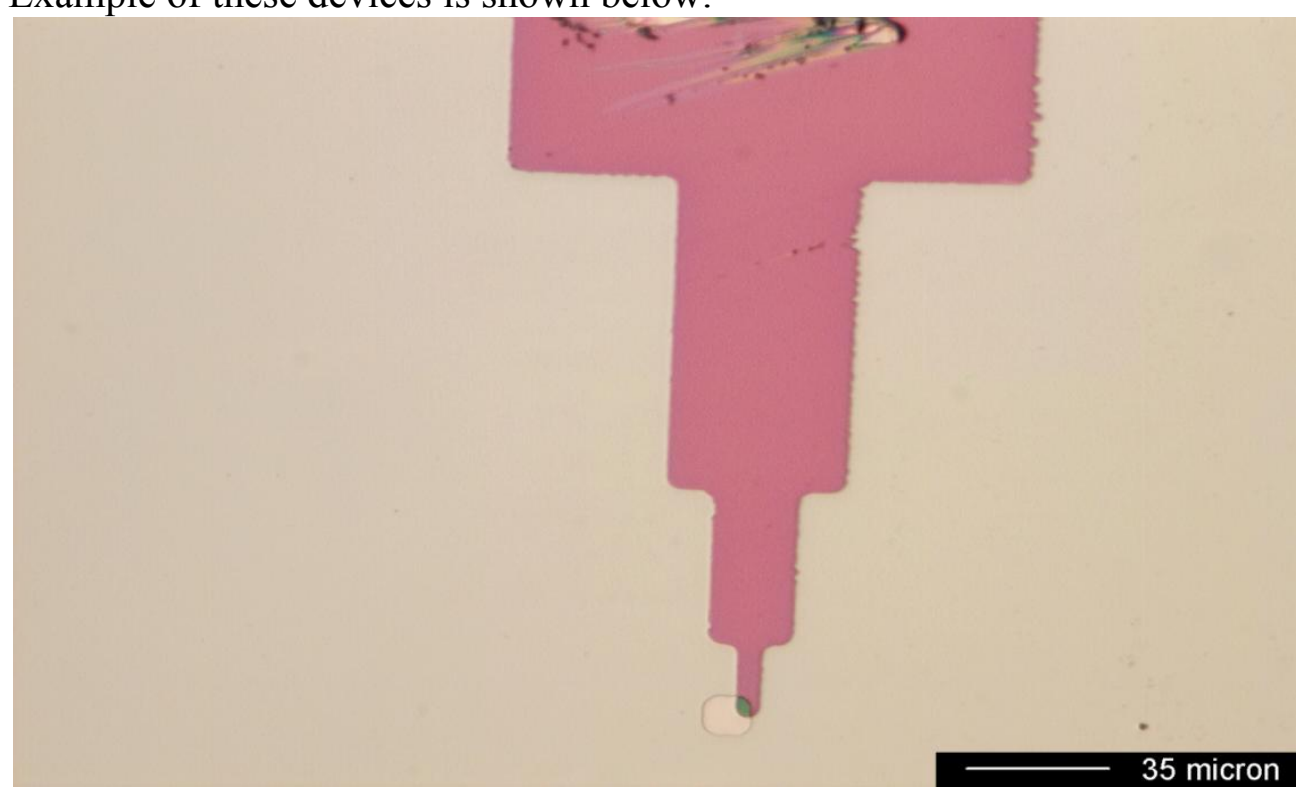

f. CMOS compatible devices with a top continuous Ag film, whose performance showed an increase in the device resistance with an increase in the corresponding radiation dose. While the data sets up to now have not clearly demonstrated that we can induce photo-diffusion by radiation exposure in a controlled manner, but some of the results suggest that properly designed devices may make effective radiation sensors. The data suggest that the $20 \%$ Ge sample will not make good sensors, while devices consisting of 30 and $40 \% \mathrm{Ge}$ with Selenium showed some sensitivity, but were already in a low resistance state. Thus we propose that the $\mathrm{Ge}_{30} \mathrm{Se}_{70}$ samples are the most suitable composition for this type of lateral sensor. Moreover, the results strongly suggest that an optimal sensor design may be achieved by significantly reducing the thickness of the ChG film.

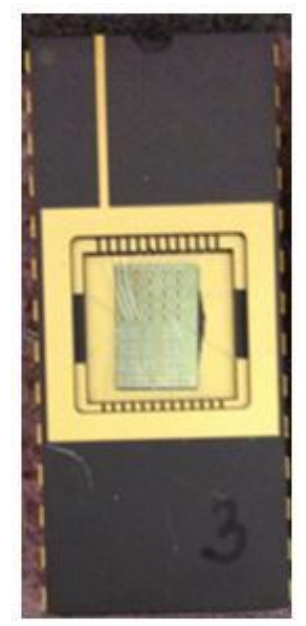




\section{Future work}

In this work, we studied radiation-sensing devices that exhibited a change in resistance due to radiation-induced silver diffusion into chalcogenide glasses. The test data demonstrated device instability, which is usual problem at creation of a new product - (i) we intend to work further to establish a stable device technology and eliminate all random factors which affect the quality of the produced devices. We studied sensor structures with three different model compositions containing 20, 30 and 40 atomic \% Ge from the Ge-S and Ge-Se systems. These compositions do not include representative from the system containing Te. There are many pros and cons for application of this system because it has bigger cross section, which is expected to have higher radiation sensitivity than the other two systems. However, this is accompanied with high polarizability, a very limited glass formation region and toxicity, which were the primary reasons that we started the research utilizing other systems. However, (ii) now we are ready to study also the system containing Te and this is what we will attempt in the near future. (iv) The irradiation results indicate that thickness and composition of ChG material play major role in the radiation induced Ag doping process and simultaneously influences the sensor performance characteristics and we will explore this in further details. The various devices studied in this research project have been fabricated using current semiconductor processes and are easily embeddable in current CMOS industry thus creating an effective, inexpensive and viable next generation radiation sensors. (v) Hence, one of the next steps in our research is creating of suitable integrated circuits for particular applications. Once an appropriate integrated circuit has been created, these various sensors can be embedded onto the chip and used for any myriad of radiation sensing purposes such as detecting radiation emitted from nuclear wastes or for personal dosimetric uses.

\section{REFERENCES}

[1] S. R. Elliott and A. Zakery, "An Introduction to Chalcogenide Glasses," in Optical Nonlinearities in Chalcogenide Glasses and their Applications. vol. 135: Springer Berlin Heidelberg, 2007, pp. 1-28.

[2] Y. Utsugi and Y. Mizushima, "Photostructural change in the Urbach tail in chalcogenide glasses," Journal of Applied Physics, vol. 51, pp. 1773-1779, 1980.

[3] M. Mitkova and D. P. Butt, "Chalcogenide Glass Ionizing Radiation Sensor," US Patent Application 20130082185.

[4] X. Feng, W. J. Bresser, and P. Boolchand, "Direct Evidence for Stiffness Threshold in Chalcogenide Glasses," Physical Review Letters, vol. 78, pp. 4422-4425, 1997.

[5] P. Boolchand, Insulating and Semiconducting Glasses: World Scientific, 2000.

[6] F. Xia, S. Baccaro, D. Zhao, M. Falconieri, and G. Chen, "Gamma ray irradiation induced optical band gap variations in chalcogenide glasses," Nuclear Instruments and Methods in Physics Research Section B: Beam Interactions with Materials and Atoms, vol. 234, pp. 525-532, 2005.

[7] A. Kovalskiy, H. Jain, A. C. Miller, R. Y. Golovchak, and O. I. Shpotyuk, "A Study of Reversible $\gamma$-Induced Structural Transformations in Vitreous $\mathrm{Ge}_{23.5} \mathrm{Sb}_{11.8} \mathrm{~S}_{64.7}$ by HighResolution X-ray Photoelectron Spectroscopy," The Journal of Physical Chemistry B, vol. 110, pp. 22930-22934, 2006/11/01 2006.

[8] C. A. Angell, K. L. Ngai, G. B. McKenna, P. F. McMillan, and S. W. Martin, "Relaxation in glassforming liquids and amorphous solids," Journal of Applied Physics, vol. 88, pp. 3113-3157, 2000. 
[9] E. I. Kamitsos, J. A. Kapoutsis, G. D. Chryssikos, G. Taillades, A. Pradel, and M. Ribes, "Structure and Optical Conductivity of Silver Thiogermanate Glasses," Journal of Solid State Chemistry, vol. 112, pp. 255-261, 1994.

[10] T. Kawaguchi, S. Maruno, and S. R. Elliott, "Optical, electrical, and structural properties of amorphous Ag-Ge-S and Ag-Ge-Se films and comparison of photoinduced and thermally induced phenomena of both systems," Journal of Applied Physics, vol. 79, pp. 9096-9104, 1996.

[11] A. Feltz, H. Aust, and A. Blayer, "Glass formation and properties of chalcogenide systems XXVI: Permittivity and the structure of glasses $\mathrm{As}_{\mathrm{x}} \mathrm{Se}_{1-\mathrm{x}}$ and $\mathrm{Ge}_{\mathrm{x}} \mathrm{Se}_{1-\mathrm{x}}$," Journal of Non-Crystalline Solids, vol. 55, pp. 179-190, 1983.

[12] V. K. Kudoyarova, "The effect of DC electric field on Ag migration in glassy Ge2S3," Journal of Non-Crystalline Solids, vol. 90, pp. 593-596, 1987.

[13] M. Mitkova, I. Iliev, V. Boev, and T. Petkova, "Influence of an electrical field on optical recording in chalco-halide glasses," Journal of Non-Crystalline Solids, vol. 227-230, Part 2, pp. 748-751, 1998.

[14] M. Popescu, Non-crystalline chalcogenides. Dordrecht; Boston: Kluwer Academic Publishers, 2000.

[15] A. V. Kolobov, Photo-induced metastability in amorphous semiconductors: Wiley-VCH, 2006.

[16] A. V. Kolobov and S. R. Elliott, "Photodoping of amorphous chalcogenides by metals," Advances in Physics, vol. 40, pp. 625-684, 1991/10/01 1991.

[17] M. Frumar and T. Wagner, "Ag doped chalcogenide glasses and their applications," Current Opinion in Solid State and Materials Science, vol. 7, pp. 117-126, 2003.

[18] T. Kawaguchi, S. Maruno, and S. R. Elliott, "Optical, electrical, and structural properties of amorphous Ag--Ge--S and Ag--Ge--Se films and comparison of photoinduced and thermally induced phenomena of both systems," Journal of Applied Physics, vol. 79, pp. 9096-9104, 1996.

[19] S. P. Thermadam, S. Bhagat, T. Alford, Y. Sakaguchi, M. Kozicki, and M. Mitkova, "Influence of $\mathrm{Cu}$ diffusion conditions on the switching of $\mathrm{Cu}-\mathrm{SiO}<\mathrm{sub}>2</$ sub $>$-based resistive memory devices," Thin Solid Films, vol. 518, pp. 3293-3298, 2010.

[20] M. N. Kozicki, "Personal electronic dosimeter," Google Patents, 1996.

[21] M. N. Kozicki and M. Mitkova, "Mass transport in chalcogenide electrolyte filmsmaterials and applications," Journal of Non-Crystalline Solids, vol. 352, pp. 567-577, 2006.

[22] M. Mitkova and M. Kozicki, "Silver incorporation in Ge-Se glasses used in programmable metallization cell devices," Journal of Non-Crystalline Solids, vol. 299, pp. 1023-1027, 2002.

[23] T. Kawaguchi and S. Maruno, "Photoinduced surface deposition of metallic silver in Ag-As-S glasses," Journal of Applied Physics, vol. 77, pp. 628-634, 1995.

[24] T. Kawaguchi, S. Maruno, and S. Elliott, "Compositional dependence of the photoinduced surface deposition of metallic silver in $\mathrm{Ag} \square$ As $\square$ S glasses," Journal of Non-Crystalline Solids, vol. 211, pp. 187-195, 1997. 


\section{PATENTS, PAPERS PUBLISHED AND CONFERENCE PARTICIATIONS}

\section{Patent}

Inventors: Maria Mitkova, Darryl P. Butt

Title and Patent Number: Chalcogenide Glass Ionizing Radiation Sensor, US Patent Application 20130082185

Abstract: A chalcogenide glass radiation sensor comprising a chalcogenide glass layer coupled to at least two electrodes and a metal source, and a method using the same are disclosed. The chalcogenide glass layer has a resistivity and the at least two electrodes are configured to facilitate the measurement of the resistivity of the chalcogenide glass layer. The coupling of the metal source and the chalcogenide glass layer is such that the resistivity of the chalcogenide glass layer changes upon exposure to ionizing radiation. The metal source is configured to be external to an electric field that may form between the at least two electrodes as the resistivity of the chalcogenide glass layer is measured.

\section{Publications}

1) Chen, P., Ailavajhala, M., Mitkova, M., Tenne, D., Esqueda, I. S., \& Barnaby, H. "Structural study of Ag-Ge-S solid electrolyte glass system for resistive radiation sensing." Microelectronics and Electron Devices (WMED), 2011 IEEE Workshop on. IEEE, 2011.

2) Ailavajhala, Mahesh, Maria Mitkova, and Darryl P. Butt. "Simulation and process flow of radiation sensors based on chalcogenide glasses for in situ measurement capability." physica status solidi (c) 9.12 (2012): 2415-2419.

3) Nesheva, D., Ailavajhala, M., Chen, P., Tenne, D. A., Barnaby, H., Mitkova, M., "Studies of Gamma Radiation Induced effects in Ge-Rich Chalcogenide Thin Films." Proceedings of the RAD 2012. pp. 19-22.

4) Mitkova, M., Chen, P., Ailavajhala, M., Butt, D.P., Tenne, D.A., Barnaby, H., Esqueda, I.S., "Gamma Ray Induced Structural Effects in Bare and Ag Doped Ge-S Thin Films for Possible Sensor Applications.” J. Non Cryst. Sol. 2013

5) Gonzalez-Velo, Y., H. J. Barnaby, A. Chandran, D. R. Oleksy, P. Dandamudi, M. N. Kozicki, K. E. Holbert, M. Mitkova, M. Ailavajhala, and P. Chen. "Effects of Cobalt-60 Gamma-Rays on GeSe Chalcogenide Glasses and Ag/Ge-Se Test Structures." Nuclear Science, IEEE Transactions on 59, no. 6 (2012): 3093-3100

6) Gonzalez-Velo, Y., H. J. Barnaby, M. N. Kozicki, P. Dandamudi, A. Chandran, K. Holbert, M. Mitkova and M. Ailavajhala "Total-Ionizing-Dose Effects on the Switching Characteristics of Chalcogenide $\left(\mathrm{Ge}_{30} \mathrm{Se}_{70}\right)$ Programmable Metallization Cells" Nuclear Science, IEEE Transactions on accepted

7) P. Dandamudi, M. N. Kozicki, H. J. Barnaby, Y. Gonzalez-Velo, K. E. Holbert, and M. Mitkova, Sensors Based on Radiation-Induced Diffusion of Silver in Germanium Selenide Glasses, Nuclear Science, IEEE Transactions on accepted. 
8) Y. Sakaguchi, H. Asaoka, Y. Uozumi, Y. Kawakita, T. Ito, M. Kubota, D. Yamazaki, K. Soyama, M. Ailavajhala and M. Mitkova "Dynamics of silver photo-diffusion in $\mathrm{Ag} / \mathrm{Ge}_{\mathrm{x}} \mathrm{S}_{1-\mathrm{x}}(\mathrm{x}=0.2$ and 0.4$)$ films investigated by neutron reflectivity" Canadian Journal of Physics (2013) (submitted)

9) Maria Mitkova, Mahesh Ailavajhala, Darryl P. Butt, Hugh Barnaby, Yago Gonzalez Velo, Christian Poweleit "Material Study and Device characterization of Ge-rich Chalcogenide films for sensing Nuclear Wastes" Proceedings of $3^{\text {rd }}$ International Conference on Research Frontiers in Chalcogen Cycle Science \& Technology (2013) (Submitted)

10) Maria Mitkova, Mahesh Ailavajhala, Darryl P. Butt, Hugh Barnaby, Yago Gonzalez Velo and Christian Poweleit "Investigation of the Structure and Performance of CMOS Compatible Lateral Radiation Sensors using Thin Film Chalcogenide Glasses" Canadian Journal of Physics (2013) (submitted)

\section{Conference Participations}

1) Mahesh Ailavajhala, Ping Chen, Hugh Barnaby, Maria Mitkova, Darryl Butt. "Radiation Induced Effects in Ag Doped Ge-Se Films”, Oral presentation, MRS Spring 2011, San Francisco. Symposium U (April 27, 2011)

2) Mahesh Ailavajhala, Ping Chen, Hugh Barnaby, Maria Mitkova, Darryl Butt. "Radiation Induced Effects in Ag Doped Ge-Se Films", Poster Presentation, Workshop on Microelectronics and Electron Devices, Boise, ID (April 22, 2011)

3) Ping Chen, Mahesh Ailavajhala, Dmitri Tenne, Ivan S. Esqueda, Hugh Barnaby, Maria Mitkova. "Structural Details and Structural Engineering of GeS Chalcogenide Glasses for Resistive Radiation Sensor Device.”, Oral presentation, MRS Spring 2011, San Francisco. Symposium K (April 29, 2011)

4) Chen, P., Ailavajhala, M., Mitkova, M., Tenne, D., Esqueda, I. S., \& Barnaby, H. "Structural study of Ag-Ge-S solid electrolyte glass system for resistive radiation sensing.", Oral Presentation, Workshop on Microelectronics and Electron Devices, Boise, ID (April 22, 2011)

5) Mahesh Ailavajhala, Ping Chen, Dmitri Tenne, Hugh Barnaby, David Oleski, Darryl P. Butt, Maria Mitkova. "Gamma-Ray Induced Structural and Electrical Changes in Ag Doped Ge-Se Thin Films.” Oral Presentation, MRS Spring 2012, San Francisco. Symposium II (April 12, 2012)

6) Mahesh Ailavajhala, Maria Mitkova, Darryl Butt. "Simulation and Process Flow of Radiation Sensors based on Chalcogenide Glasses for insitu measurements" Poster Presentation, MRS Spring 2012, San Francisco. Symposium CCC (April 11,2012)

7) Mahesh Ailavajhala, Ping Chen, Maria Mitkova, Darryl Butt, David Olesky, Yago G. Velo, Hugh Barnaby. "Dependence of the Structure on Performance of Chalcogenide 
Glass Based Radiation Sensors." Poster Presentation, Workshop on Microelectronics and Electron Devices, Boise, ID (April 20, 2012)

8) Maria Mitkova, Ping Chen, Mahesh Ailavajhala, Darryl Butt, Dmitri Tenne, Hugh Barnaby, Ivan Esqueda. "Gamma Radiation induced silver diffusion in Ge-Chalcogenide glasses.", oral presentation, The International Symposium on Non Oxide and New Optical Glasses (ISNOG) 2012, Saint-Malo, France (July 3, 2012)

9) Maria Mitkova, Mahesh Ailavajhala, Darryl P. Butt. "Simulation and Process flow of radiation sensors based on chalcogenide glasses for insitu measurement capability" Oral Presentation, International Conference on Optical, Optoelectronic and Photonic Materials and Applications (ICOOPMA ) 2012, Nara, Japan (June 4, 2012)

10) Nesheva, D., Ailavajhala, M., Chen, P., Tenne, D. A., Barnaby, H., Mitkova, M., "Studies of Gamma Radiation Induced effects in Ge-Rich Chalcogenide Thin Films." Oral presentation, RAD 2012, Nís, Serbia (April, 2012)

11) D. Oleksy, H. Barnaby, M. Kozicki, M. Mitkova, M. Ailavajhala, P. Chen. "Basic Radiation-Effects Mechanisms in Chalcogenide Based nanoionic structures" Poster presentation, Government Microcircuit Applications \& Critical Technology Conference (GOMAC), Las Vegas, NV (March 22, 2012)

12) Mahesh Ailavajhala, Ping Chen, Maria Mitkova, Darryl Butt, David Olesky, Yago G. Velo, Hugh Barnaby. "Dependence of the Structure on Performance of Chalcogenide Glass Based Radiation Sensors." Poster Presentation, Idaho NSF EPSCoR \& Idaho NASA EPSCoR Annual Meeting, Boise, ID (October 2, 2012)

13) Mahesh Ailavajhala, Hugh Barnaby, Yago G. Velo, Maria Mitkova, Darryl Butt. "Application of Ion Transport in Chalcogenide Glasses for Radiation Sensing." Poster presentation, International Material Institute- New Functionality of Glasses (IMI-NFG), Siracusa, Italy (January 12, 2013) (Best Poster Award)

14) Mahesh Ailavajhala, Ping Chen, Dmitri Tenne, Hugh Barnaby, Yago G. Velo, Maria Mitkova, Darryl Butt. "Simulation and Structural Analysis of Gamma Radiation Induced Ag Diffusion in Ge-Se Thin Films and Devices." Oral presentation, MRS Spring 2013, San Francisco. Symposium WW (April 2, 2013)

15) Gonzalez-Velo, Y., H. J. Barnaby, M. N. Kozicki, P. Dandamudi, A. Chandran, K. Holbert, M. Mitkova and M. Ailavajhala "Total-Ionizing-Dose Effects on the Switching Characteristics of Chalcogenide $\left(\mathrm{Ge}_{30} \mathrm{Se}_{70}\right)$ Programmable Metallization Cells" Poster presentation, NSREC 2013, San Francisco (July 8-12, 2013) 